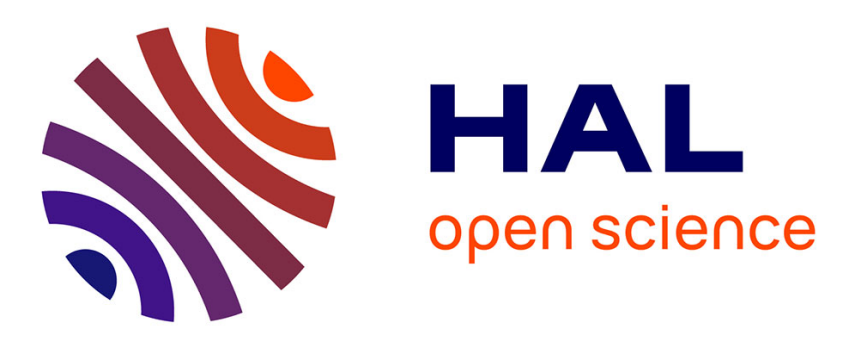

\title{
Phase field model for the martensitic transformation: comparison of the Voigt/Taylor and Khachaturyan approach
}

Simon David Schmidt, Kais Ammar, Wolfgang Dornisch, Samuel Forest, Ralf Müller

\section{To cite this version:}

Simon David Schmidt, Kais Ammar, Wolfgang Dornisch, Samuel Forest, Ralf Müller. Phase field model for the martensitic transformation: comparison of the Voigt/Taylor and Khachaturyan approach. Continuum Mechanics and Thermodynamics, 2021, 10.1007/s00161-021-01007-1 . hal03243118

\author{
HAL Id: hal-03243118 \\ https://hal.science/hal-03243118
}

Submitted on 16 Oct 2021

HAL is a multi-disciplinary open access archive for the deposit and dissemination of scientific research documents, whether they are published or not. The documents may come from teaching and research institutions in France or abroad, or from public or private research centers.
L'archive ouverte pluridisciplinaire $\mathbf{H A L}$, est destinée au dépôt et à la diffusion de documents scientifiques de niveau recherche, publiés ou non, émanant des établissements d'enseignement et de recherche français ou étrangers, des laboratoires publics ou privés. 


\title{
Simon David Schmidt $₫$ • Kais Ammar • Wolfgang Dornisch • Samuel Forest · Ralf Müller \\ Phase field model for the martensitic transformation: comparison of the Voigt/Taylor and Khachaturyan approach
}

Received: 23 July 2020 / Accepted: 19 March 2021

(C) The Author(s), under exclusive licence to Springer-Verlag GmbH Germany, part of Springer Nature 2021

\begin{abstract}
Iron and steels are allotropes, meaning they exhibit different crystal configurations. The martensitic transformation is crucial for a variety of processes, such as hardening. It is induced by a combination of undercooling and mechanical deformation. Due to the changing material properties within the phases, and due to topological changes that might occur during the transformation, a phase field approach was chosen that incorporates both the mechanical and the chemical aspect of this problem. A comparison of the Voigt/Taylor approach to the Khachaturyan approach within a multi-variant phase field modeling of the martensitic transformation including a chemical and a mechanical energy contribution is presented in this paper. The model was implemented in the finite element codes FEAP and Z-set independently. Numerical examples are given in order to highlight the features of this model.
\end{abstract}

Keywords Phase field model $\cdot$ Homogenization $\cdot$ Martensitic transformation $\cdot$ Finite elements

\section{Introduction}

Iron and steels forming crystal structures exhibit allotropes with different mechanical and chemical properties $[25,53,60]$. Here, mainly two allotropes are of interest: the face-centered cubic (fcc) austenite and the bodycentered cubic (bcc) martensite. While the austenitic phase is considered more ductile, the harder martensitic phase is often desirable in near-surface regions, which are subject to mechanical strain and wear. Mechanical properties and lattice constants of the allotropes have been investigated in, e.g., $[2,24,27,32,33,36]$.

Solid-solid phase transformations are of interest in the context of surface hardening [9,31]. The martensitic transformation (MT) is a diffusionless process as described in, e.g., $[1,12,13,40,50,56]$. Atom movement during the MT is restricted to the interatomic distances, which results in a fast $\left(10^{-7} \mathrm{~s}\right)$ transformation compared to diffusive processes. In [56], two basic types of diffusionless transformations are considered: a global transformation where all atoms move simultaneously and a civilian type of transformation where the atoms move individually. The MT belongs to the latter.

Communicated by Andreas Öchsner.

R. Müller · S. Schmidt $(\varangle)$

Institute of Applied Mechanics, Technische Universität Kaiserslautern, P.O. Box 3049, 67653 Kaiserslautern, Germany

E-mail: sdschmid@rhrk.uni-kl.de

K. Ammar - S. Forest

Centre des Matériaux, MINES Paristech, PSL University, CNRS UMR, 7633 Paris, France

E-mail: kais.ammar@mines-paristech.fr

W. Dornisch

Fachgebiet Statik und Dynamik, Brandenburgische Technische Universität Cottbus-Senftenberg, Konrad-Wachsmann-Allee 2, 03046 Cottbus, Germany 
In this work, three influences on the formation of the martensitic phase are admitted. First, the lattice mismatch between the austenitic parent phase and the transformed martensitic phases induces an eigenstrain. The nature of this eigenstrain can be described with the orientation relationship. Historically, the transformation mechanism proposed by Bain in [10] remains the case with the fewest transformation; however, more complicated cases are possible. Consequently, the transformation of a parent unit cell may lead to differently oriented martensitic child phases, introducing mirrored and rotated eigenstrains and thus motivating the presence of several orientation variants.

Secondly, the influence of stress on the MT is relevant. Stress can induce a phase change from the austenitic to the martensitic phase. This has been shown both experimentally [9] and theoretically [8]. The coupling of the MT with a mechanical constitutive model through the eigenstrain with the stress accounts for a stress-induced MT.

Thirdly, there is an influence of the temperature on the MT. There have been molecular dynamic calculations studying the path of the MT in dependence of temperature [43]. As a result, the free energy is crucial for the evolution of the martensite phases. In $[44,46]$ a temperature-dependent energy functional is discussed. However, the temperature diffusion and the MT live in different time frames; therefore, the transience of the temperature is neglected.

For this study, a phase field model is used. Phase field models are a versatile tool for various interfacial problems, as they introduce a mathematical regularization of the sharp interface approach, scaling all phasedependent values with an additional degree of freedom, the order parameter [41]. Therefore, they automatically account for topological changes and have been used for modeling, e.g., solidification [17,57], phase transformation in solids [7,8], fracture problems [29,34,35] and multi-phase flows [3,16,21]. Typically, the order parameter or a set of order parameters characterizes a phase's presence, such as in [29,35], or a concentration such as in $[5,16]$. Here, according to the former, a vector-valued order parameter identifies the transformed child phases. There are two main types of evolution equations: a so-called conserving evolution equation proposed by Cahn and Hillard $[15,26]$, modeling the diffusion process of a field quantity. In the case of transformation phenomena, the non-conserving evolution equation of the Ginzburg-Landau problem proposed by Allen and Cahn is suitable for diffusionless transformations $[4,14]$. The latter is used in our case.

The MT has been modeled with a variety of approaches. Multi-phase fields have been proposed by [47,52]. In those cases the phases present are identified by a set of order parameters. In contrast to multi-variant phase field approaches, such as in [46], a matrix mobility allows transformations from either one of the phases to another. Homogenization schemes for the modeling of plasticity have been employed in, e.g., [22]. Here, we consider a multi-variant phase field approach with a constant mobility and separate evolution equations for each order parameter. Consequently, the transformation between child phases is not explicitly modeled. Such a transformation is still possible where one child phase transforms to the parent phase, and the parent phase simultaneously transforms to the other child phase. In this paper, we present a multi-variant Voigt/Taylor homogenization phase field approach. We essentially start with a Khachaturyan type approach for the MT as proposed in, e.g., [46] and move toward a homogenization type approach as proposed in [5,6] for a multivariant phase field. The paper is structured as follows: First, the phase field model with a vector-valued order parameter is established, accounting for the different transformed child phases. We then motivate the energetic setup starting from the micro force balance as proposed in [19] and identify our field equations. We consider the energy to be split up additively into a chemical part and a mechanical part. As phase field models rely on interpolating phase-dependent quantities, we discuss different modes of interpolation in multi-variant phase field approaches and show how we can ensure that the partition of unity holds even if using non-linear interpolation functions. Specific regularization schemes of the energy are given in the Khachaturyan and the Voigt/Taylor approach. We demonstrate that the Khachaturyan approach can lead to unphysical interpolation behavior of the interface's elastic energy. Another methodical advantage of the Voigt/Taylor approach is the interchangeability of the child phases' constitutive behavior. The model has been implemented and solved with a monolithic finite element scheme.

\section{Phase field model}

Consider a scalar-valued order parameter $\phi$ living on $[0,1]$, where $\phi=0$ if the untransformed or parent phase is assumed and $\phi=1$ where the transformed or child phase is assumed. Let $f(\phi, \underline{\nabla} \phi)$ be an energy density for the bulk phases and the interface in dependence of a phase field parameter $\phi$ and its gradient $\underline{\nabla} \phi$. The order parameter $\phi$ interpolates the energy $f$ such that $f(0, \underline{0})$ is the bulk energy for the parent phase, and $f(1, \underline{0})$ is the bulk energy for the transformed phase. The interface is the region where $0<\phi<1$. 
The presence of more than two phases, for instance when considering several orientation relationships of the martensitic transformation (MT), motivates the introduction of a vector-valued order parameter $\phi \in \mathbb{R}^{n}$. The representation in summation convention $\phi=\phi_{\alpha} \underline{e}_{\alpha}=\left[\phi_{1}, \ldots \phi_{n}\right]^{\top}$ reveals the components $\phi_{\alpha}$, where $\alpha$ ranges from 1 to the number of martensitic orientation variants $n$. The $\alpha$-th child phase is present when $\phi=\underline{e}_{\alpha}$, where $\underline{e}_{\alpha}$ is a unit orthogonal basis vector in $\mathbb{R}^{n}$. In the following, Greek indices $(\alpha, \beta, \gamma, \ldots)$ live on $[0,1, \ldots, n]$, while Latin indices $(i, j, k, \ldots)$ denote spatial components. The parent phase is present whenever $\phi=\underline{0}$, as this denotes the absence of all $n$ child phases together. The components in the vector-valued order parameter are independent. The parent phase is identified with $\phi_{0}$. Locally, the sum of the chemical components $\phi_{\alpha}$ is required to be constant [41]; therefore,

$$
\phi_{0}+\sum_{\alpha=1}^{n} \phi_{\alpha}=1
$$

has to hold, where $\phi_{0}=1$ if only the parent phase is present. Note at this point, that $\phi_{0}$ is not a component of $\phi$. Therefore, following from (1), the order parameter identifying the parent phase is dependent $\phi_{0}=1-\sum_{\alpha=1}^{n} \phi_{\alpha}$. The energy density $f$ is a function of the individual vector-valued order parameter $\phi$ and their gradients $\underline{\nabla} \otimes \phi$. The energy density has to assume its respective value $f_{\alpha}$ for each individual bulk phase. Vanishing gradient terms $\underline{\nabla} \phi_{\alpha}=\underline{0}$ in the bulk lead to

$$
f\left(\underline{\phi}=\underline{e}_{\alpha}, \underline{\nabla} \otimes \underline{\phi}=\underline{\mathbf{0}}\right)=f_{\alpha}, \quad \text { for all } \alpha \in[0,1, \ldots, n] .
$$

We define $\underline{e}_{0}=\underline{0}$, such that $\underline{\phi}=\underline{0}$ and $f_{0}$ represents the bulk energy in the parent phase. The order parameter is non-conserved. Therefore, the evolution of the order parameter component $\phi_{\alpha}$ with respect to time $t$ is a Ginzburg-Landau time-dependent evolution equation

$$
\frac{\partial \phi_{\alpha}}{\partial t}=-M \frac{\delta E}{\delta \phi_{\alpha}}=-M\left[\frac{\partial f}{\partial \phi_{\alpha}}-\underline{\nabla} \frac{\partial f}{\partial\left(\underline{\nabla} \phi_{\alpha}\right)}\right],
$$

which is derived from the minimization of the local free energy $E=\int_{V} f \mathrm{~d} V$. In the multi-variant field approach presented here, only transformations from the parent phase to the child phases are modeled. In (3) a vector-valued mobility could be introduced [52]; however, all orientation variants are considered to be equally stable without preferential treatment. Therefore, the scalar proportional constant or mobility $M \geq 0$ scaling the kinematics of the evolution is assumed to be constant.

\section{Energetic setup}

We motivate the balance and evolution equations by the balance of micro forces following [19]. Let $\dot{e}$ be the rate of the energy density,

$$
\dot{e}=-\pi_{\alpha} \dot{\phi}_{\alpha}+\xi_{\alpha i} \dot{\phi}_{\alpha, i}+\sigma_{i j} \dot{\varepsilon}_{i j}
$$

where summation convention applies. The strain tensor due to the displacements $\underset{\sim}{\varepsilon}=\varepsilon_{i j} \underline{e}_{i} \otimes \underline{e}_{j}$ is symmetric in linear small strain theory. The small strain

$$
\underset{\sim}{\varepsilon}={\underset{\sim}{\varepsilon}}^{\mathrm{e}}+{\underset{\sim}{\varepsilon^{*}}}^{*}
$$

is the sum of an elastic strain ${\underset{\sim}{\mathcal{E}}}^{\mathrm{e}}$ and an inelastic strain. In general, the inelastic strain contains, e.g., the plastic strain and any transformational parts. In the present work, it is restricted to the transformation strain $\varepsilon^{*}$ only; plasticity is not considered. As a consequence of the balance of linear and angular momentum, the stress tensor $\underset{\sim}{\sigma}=\sigma_{i j} \underline{e}_{i} \otimes \underline{e}_{j}$ is symmetric. Due to the absence of mass transport, the change of the entropy $\dot{s}$ is related with

$$
T \dot{s} \geq 0 .
$$

Assuming the flux of the free energy density $\dot{f}=\dot{e}-T \dot{s}$, we obtain the Clausius-Duhem inequality

$$
-\dot{f}-\pi_{\alpha} \dot{\phi}_{\alpha}+\xi_{\alpha i} \dot{\phi}_{\alpha, i}+\sigma_{i j} \dot{\varepsilon}_{i j} \geq 0 \text {. }
$$


Introducing the total derivative of $f$ into (7) yields

$$
-\left(\pi_{\alpha}-\frac{\partial f}{\partial \phi_{\alpha}}\right) \dot{\phi}_{\alpha}+\left(\xi_{\alpha i}-\frac{\partial f}{\partial \phi_{\alpha, i}}\right) \dot{\phi}_{\alpha, i}+\left(\sigma_{i j}-\frac{\partial f}{\partial \varepsilon_{i j}^{\mathrm{e}}}\right) \dot{\varepsilon}_{i j}^{\mathrm{e}} \geq 0,
$$

identifying the equations of state. The energy density is assumed to be additively split up into an elastic part $f_{\mathrm{u}}$ due to the local elastic strain ${\underset{\sim}{\mathrm{e}}}^{\mathrm{e}}$, and a chemical free energy $f_{\text {ch }}$ due to the energy release of the phase transformation

$$
f\left(\underline{\phi}, \underline{\nabla} \otimes \underline{\phi}, \underline{\varepsilon}^{\mathrm{e}}\right)=f_{\mathrm{u}}\left(\underline{\varepsilon}^{\mathrm{u}}, \underline{\phi}\right)+f_{\mathrm{ch}}(\underline{\phi})+f_{\mathrm{grad}}(\underline{\nabla} \otimes \underline{\phi}) .
$$

The matrix $\underline{\nabla} \otimes \phi$ is a concatenation of the vectors $\underline{\nabla} \phi_{\alpha}$. The gradient term $f_{\text {grad }}$ vanishes for the bulk. It is therefore only present on the interface, where the gradients of the individual order parameters components $\underline{\nabla} \phi_{\alpha}$ are nonzero. The gradient term is

$$
f_{\text {grad }}(\underline{\nabla} \otimes \underline{\phi})=\frac{\alpha_{\text {grad }}}{2}(\underline{\nabla} \otimes \underline{\phi}):(\underline{\nabla} \otimes \underline{\phi}),
$$

where (:) denotes the double contraction. The chemical free energy $f_{\text {ch }}$ is temperature dependent; however, in this work a constant temperature $T$ is considered. We relate the chemical free energy to a normalized double well or Landau polynomial

$$
\begin{aligned}
f_{\text {ch }}(\underline{\phi}) & =\beta_{\text {ch }} f_{\mathrm{L}}(\underline{\phi}), \text { with } \\
f_{\mathrm{L}}(\underline{\phi}) & =\frac{1}{2} A \sum_{\alpha=1}^{n}\left[\phi_{\alpha}^{2}\right]-\frac{1}{3} B \sum_{\alpha=1}^{n}\left[\phi_{\alpha}^{3}\right]+\frac{1}{4} C\left[\sum_{\alpha=1}^{n}\left[\phi_{\alpha}^{2}\right]\right]^{2}+1 .
\end{aligned}
$$

The Landau polynomial is 1 when in the parent phase, and it vanishes when in one of the transformed phases $\alpha=1, \ldots, n$. Its first derivatives with respect to the order parameter components $\phi_{\alpha}$ are assumed to vanish when either in the parent or the transformed phases which renders $B$ and $C$ dependent on $A$ [59]

$$
B=12+3 A, \quad \text { and } C=B-A .
$$

The parameter $A$ can be related to the temperature $T$ [44]. The proportional constants $\alpha_{\text {grad }}$ and $\beta_{\text {ch }}$ can be chosen to represent measures for the interfacial width $L$ and the energy density $G$, which is attributed to the interface

$$
\alpha_{\text {grad }}=\kappa_{\text {grad }} G L \quad \text { and } \quad \beta_{\mathrm{ch}}=\kappa_{\text {sep }} \frac{G}{L},
$$

where $\kappa_{\text {grad }}$ and $\kappa_{\text {sep }}$ are calibration constants. The elastic energy density $f_{\mathrm{u}}$ is a function of the elastic strain ${\underset{\sim}{\mathrm{e}}}^{\mathrm{e}}$. In a small strain setting, the total strain is assumed to be additively split up according to (5). The transformation strain $\mathcal{\sim}^{*}$ is modeled as a function of the phases present. It is not further specified at this point. Let the elastic energy density within a phase $\alpha=0, \ldots, n$ be

$$
f_{\mathrm{u}_{\alpha}}=\frac{1}{2} \varepsilon_{\alpha}^{\mathrm{e}}: \underset{\approx \alpha}{\mathbf{C}}: \varepsilon_{\sim \alpha}^{\mathrm{e}}
$$

where the elastic strain ${\underset{\sim}{\alpha}}_{\alpha}^{\mathrm{e}}$ and the material constants $\underset{\sim \alpha}{\mathbf{C}}$ depend on the phase. The choice of $f_{\mathrm{u}}$ has to conform to (2). This is apparent when regarding one distinct phase $\alpha$ in view of (11)

$$
f\left(\underline{e}_{\alpha}, \underline{\nabla} \otimes \underline{\phi}=\underline{\mathbf{0}}\right)=f_{\mathrm{u}}\left(\underline{\varepsilon}^{\mathrm{e}}, \underline{e}_{\alpha}\right)+f_{\mathrm{ch}}\left(\underline{e}_{\alpha}\right) \Rightarrow f_{\mathrm{u}}\left(\underline{e}_{\alpha}, \underline{\varepsilon}^{\mathrm{e}}\right)=f_{\mathrm{u}_{\alpha}} .
$$

For the mechanical part of this problem, the balance of linear and angular momentum holds, and the stress can be identified using (7)

$$
\underline{\nabla} \cdot \underset{\sim}{\sigma}=\underline{0}, \text { where } \sigma=\frac{\partial f}{\partial \underline{\sim}^{e}},
$$

in the static case and in the absence of volume forces. The equations (16) and (3) constitute our field equations for the mechanical and the phase field part, respectively. 


\section{Interpolation}

In the previous sections, assumptions about the total free energy within a phase $\alpha$ in (2), the chemical free energy in dependence of the phase setup $\phi$ in (11), and the evolution of the components of the order parameter $\phi_{\alpha}$ in (3) have been made. This leaves the elastic energy on the interface yet to be determined. No matter which method is used, phase field approaches rely on interpolating values across the interface. Consider a quantity $a$, which may be a scalar, vector, matrix or tensor supporting scalar multiplication, and a scalar interpolation function $h(\phi)$. The value $a$ is a function of the order parameter $\phi$ and assumes $a_{\alpha}$ when in the $\alpha$-th bulk phase

$$
a\left(\underline{\phi}=\underline{e}_{\alpha}\right)=a_{\alpha}
$$

This is analogous with (2). The quantity $a$ can be thought of as a placeholder for the effective material parameters as in the Khachaturyan approach (Sect. 5), or for the elastic energy or the stress as in the Voigt/Taylor model (Sect. 6). The function $h$ is continuous on $[0,1]$ and interpolates smoothly between $\phi=0$ and $\phi=1$, such that

$$
h(0)=0 \text { and } h(1)=1
$$

hold. Numerical implementations require first and second order derivatives with respect to the degrees of freedom or in our case with respect to the components of the order parameter. A possible interpolation for $a$ is the sum of the bulk phase values $a_{\alpha}$ multiplied by a phase-dependent interpolation function $h^{\alpha}(\underline{\phi})$

$$
a=\sum_{\alpha=0}^{n} h^{\alpha}(\phi) a_{\alpha} .
$$

In contrast to all other sums given previously, here we sum up from $\alpha=0,1, \ldots, n$ in order to include the parent phase $(\alpha=0)$. The interpolation function $h^{\alpha}$ is 1 when in the corresponding phase $\alpha$, and vanishes for all other phases

$$
h^{\alpha}(\underline{\phi})= \begin{cases}1, & \text { if } \underline{\phi}=\underline{e}_{\alpha} \\ 0, & \text { otherwise }\end{cases}
$$

The equation (20) only defines the boundary of $h^{\alpha}$; however, the behavior in the interface is not yet determined. The choice of $h^{\alpha}$ is not trivial. Defining the interpolation in dependence of a scalar function $h$ simplifies the implementation. A possible choice for the interpolation function is

$$
h^{\alpha}(\underline{\phi})=h_{\mathrm{an}}^{\alpha}(\underline{\phi})=h\left(\phi_{\alpha}\right),
$$

where the requirements for the scalar interpolation function are defined in (18) and the order parameter for the parent phase $\phi_{0}$ is defined in (1). The advantage of that approach $h_{\mathrm{an}}^{\alpha}(\phi)$ is that it works analogously for all the order parameters $\phi_{\alpha}$. Furthermore, there is no case dependency for the implementation. Although this approach is simple, in the following we show that it is only valid when either using a linear $h$ or when considering at most two different phases. However, in the general case, this interpolation is unphysical and can lead to wrong solutions and numerical instabilities.

Consequently, rather than choosing $h^{\alpha}$ analogously for all the phases, we introduce a case dependency for the parent phase. The equations read

$$
h^{\alpha}(\underline{\phi})=h_{\mathrm{if}}^{\alpha}\left(\underline{\phi)}= \begin{cases}1-\sum_{\alpha=1}^{n} h\left(\phi_{\alpha}\right) & \text { if } \alpha=0 \\ h\left(\phi_{\alpha}\right) & \text { if } \alpha>0 .\end{cases}\right.
$$

Consider a linear scalar function $h(\phi)$, where (18) still holds. In that case, the analogous interpolation function $h_{\mathrm{an}}^{\alpha}$ is equal to the interpolation with a case dependency $h_{\mathrm{if}}^{\alpha}$. As $h_{\mathrm{if}}^{\alpha}$ is equivalent to $h_{\mathrm{an}}^{\alpha}$, when regarding the child phases $(\alpha=1 \ldots n)$, it is sufficient to prove $h_{\mathrm{if}}^{0}=h_{\mathrm{an}}^{0}$. Therefore,

$$
h_{\mathrm{an}}^{0}(\underline{\phi})=h\left(\phi_{0}\right)=h\left(1-\sum_{\alpha=1}^{n} \phi_{i}\right) \stackrel{h(\phi)=\phi}{=} h(1)-\sum_{\alpha=1} h\left(\phi_{0}\right)=h_{\mathrm{if}}^{0} \underline{(\phi)} .
$$




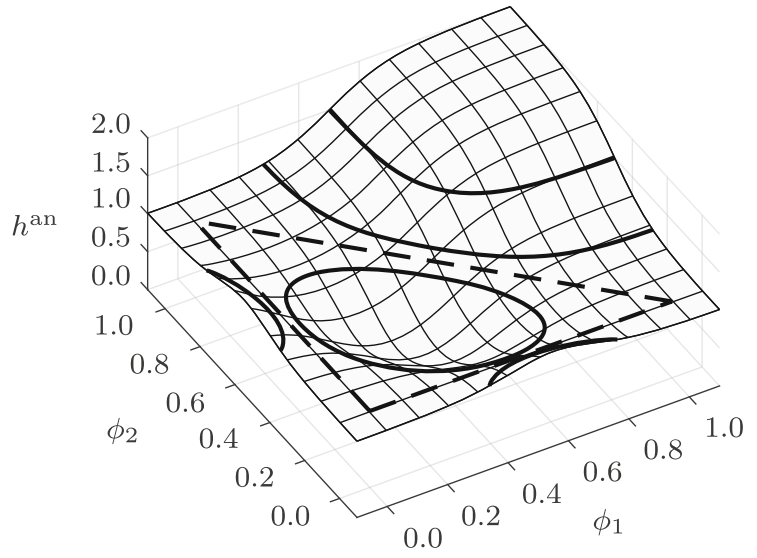

(a)

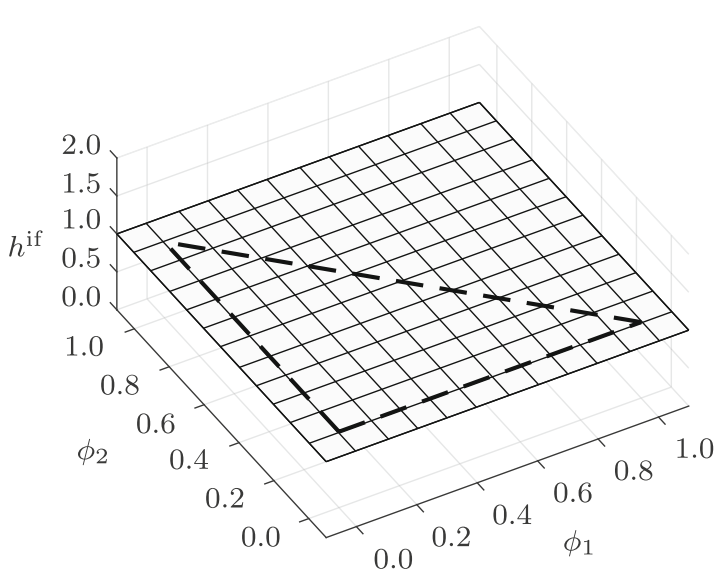

(b)

Fig. 1 Interpolation of a constant $a_{\alpha}=a_{\mathrm{ct}}=1$ with $h=h_{\text {tanh }}$ using $h_{\text {an }}$ (a) and using $h_{\text {if }}$ (b). The thick solid lines indicate contours. The dashed lines mark the boundary of the admissible region where (1) holds

That means, if all $h^{\alpha}=h_{\mathrm{if}}^{\alpha}$ is a valid choice, the analogous approach in the linear case of $h$ is valid as they both coincide for any number of phases present. We will discuss the validity of $h_{\mathrm{if}}^{\alpha}$ after looking at the case of two phases $(n=1)$. Here, function $h$ is assumed to be point symmetric with

$$
h(\phi)=1-h(1-\phi),
$$

which is valid for a wide range of interpolation functions, a small selection is given in (35), (36), and (37). Considering two phases, the interpolation function for the parent phase reads

$$
\begin{aligned}
& h_{\mathrm{an}}^{0}(\underline{\phi})=h\left(\phi_{0}\right)=h\left(1-\sum_{\alpha=1}^{n} \phi_{\alpha}\right) \stackrel{\text { symmetry }}{=} \\
& 1-h\left(\sum_{\alpha=1}^{n} \phi_{\alpha}\right) \stackrel{n=1}{=} 1-h\left(\phi_{1}\right)=h_{\mathrm{if}}^{0}(\underline{\phi}),
\end{aligned}
$$

which is equivalent to the case-dependent choice of $h_{\mathrm{if}}^{\alpha}$. However, in general number of phases, the analogous interpolation in (21) is not equivalent to the case-dependent approach given in (22), even if assuming symmetry for $h$. In general, this can lead to mixtures which exceed the values in the bulk phases such that drop-offs and local minima can occur. We assume a system of $n+1$ phases identified by $\phi_{0}, \phi_{1}, \ldots, \phi_{n}$, and show if only two phases $(\alpha$ and $\beta$ ) are present locally that the two cases presented here are equivalent. However, when adding a third phase to the mixture, we get a different behavior. First, define a vector $\phi$, where only two phases are present. Keeping (1) in mind, this vector must be of the form

$$
\underline{\phi}_{I I}=\phi_{\alpha} \underline{e}_{\alpha}+\left(1-\phi_{\beta}\right) \underline{e}_{\beta}
$$

In view of (21), we can use the symmetry in (24) to show that in this case the analogous $h_{\text {an }}^{\alpha}$ and the casedependent $h_{\mathrm{if}}^{\alpha}$ are indeed the same, meaning

$$
h_{\mathrm{an}}^{\alpha}\left(\underline{\phi}_{I I}\right)=h_{\mathrm{if}}^{\alpha}\left(\underline{\phi}_{I I}\right) .
$$

However, when adding a third phase, the two interpolation approaches differ. Fig. 1 demonstrates the interpolation for a three phase system and a symmetric $h$ for both cases if $a_{\alpha}=a_{\mathrm{ct}}=1$. The dashed lines are the boundary of the admissible region for $\phi$ and coincidentally indicate the state with at most two phases present at once. The quantity $a$ assumes the value of 1 regardless of the method chosen. Given $h_{\mathrm{if}}^{\alpha}$, depicted in Fig. 1 (b), a plane at $a=1$ is obtained. This is the expected behavior, as the mixture should not exceed the value of any of the pure phases. However, when regarding $h_{\mathrm{an}}^{\alpha}$ as in Fig. 1 (a), this leads to a drop even if the order 
parameter moves within the admissible region, but it can also lead to numerical instabilities or wrong solutions due to the extremal points outside the admissible region. Another downside of this interpolation approach is that it scales with the choice of the parameters $a_{\alpha}$, as rearranging of (19) reveals

$$
a=a_{\mathrm{ct}} \sum_{\alpha=0}^{n} h_{\mathrm{an}}^{\alpha}(\underline{\phi}) .
$$

Using the case-dependent case, the sum of the functions $h_{\text {if }}^{\alpha}$ becomes one, therefore obtaining the expected value $a_{\mathrm{ct}}$ in the interface region of more than two phases

$$
a=a_{\mathrm{ct}} \sum_{\alpha=0}^{n} h_{\mathrm{if}}^{\alpha}\left(\phi_{1}, \ldots, \phi_{n}\right)=a_{\mathrm{ct}} .
$$

Furthermore, as there are no extremal points outside the admissible region, there is no energetic incentive for the order parameter to become un-admissible. With that, the bulk phases represent energetic minima due to the chemical energy. Therefore, in order to interpolate correctly, it is required that either

1. $h$ is linear, or

2. at most two phases $(n<2)$ are present in the system at once, or

3 . the sum of the interpolation functions $h^{\alpha}\left(\phi_{1}, \ldots, \phi_{n}\right)$ becomes one.

Following from the latter, with addition to the sum of order parameters to be one (1), the partition of unity for the set of interpolation functions $h^{\alpha}$ has to hold

$$
\sum_{\alpha=0}^{n} h^{\alpha}\left(\phi_{1}, \ldots, \phi_{n}\right)=1 .
$$

This is automatically the case for any number of phases when using $h_{\text {if }}^{\alpha}(22)$. The partition of unity holds as well for a linear interpolation function as a consequence of (1) for any number of phases, or in view of (24) for at most two phases. Implementation-wise, as shown above, both cases coincide in the case of a linear $h$, not restricting the use of a linear function.

\section{Khachaturyan approach}

Up until here, simply the conformity to (2) is assumed. The behavior of the elastic energy density on the interface is not yet specified. In the following, we present the Khachaturyan and the Voigt/Taylor homogenizational approach discussing this issue. For the Khachaturyan approach [7], we closely follow [44,46]. For the interface, we suppose that (14) holds. We scale the effective material parameters with a scalar function $h$. The material moduli $\underset{\sim}{\mathbf{C}}$ and the transformational strain ${\underset{\sim}{*}}^{*}$ are

$$
\begin{gathered}
\underset{\approx}{\mathbf{C}}(\underline{\phi})=\underset{\approx 0}{\mathbf{C}} h^{0}(\underline{\phi})+\sum_{\alpha=1}^{n} \underset{\approx \alpha}{\mathbf{C}} h\left(\phi_{\alpha}\right) \text { and } \underline{\varepsilon}^{*}(\underline{\phi})=\varepsilon_{0}^{*} h^{0}(\underline{\phi})+\sum_{\alpha=1}^{n} \underline{\varepsilon}_{\alpha}^{*} h\left(\phi_{i}\right) \\
\text { where } h(0)=0, h(1)=1, \text { and } h^{0}(\underline{\phi})=1-\sum_{\alpha=1}^{n} h\left(\phi_{\alpha}\right) .
\end{gathered}
$$

By the choice of the parameters, the mechanical energy $f_{\mathrm{u}}$ is fully defined and fulfills (2):

$$
f_{\mathrm{u}}=\frac{1}{2} \mathcal{\sim}^{\mathrm{e}}: \underset{\sim}{\mathbf{C}}: \underset{\sim}{\mathcal{E}} .
$$

Regarding (33), for example the following functions can be used:

$$
\begin{aligned}
& h_{\text {linear }}(\phi)=\phi \\
& h_{\text {cubic }}(\phi)=3 \phi^{2}-2 \phi^{3}
\end{aligned}
$$



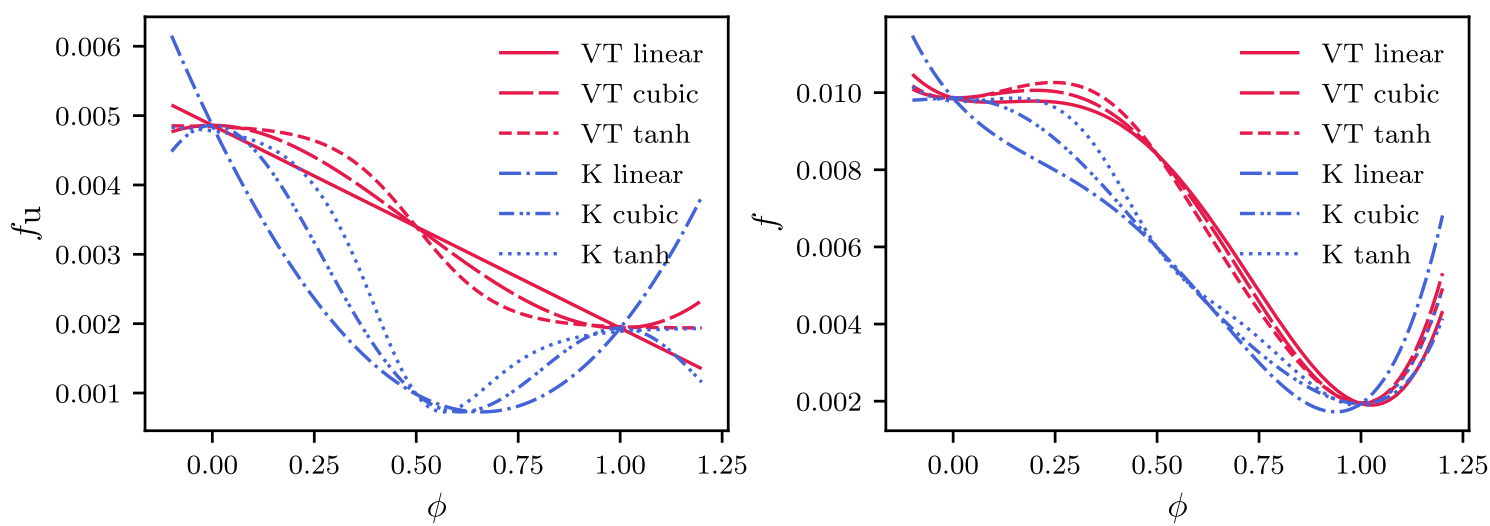

Fig. 2 Elastic energy for the Khachaturyan and the Voigt/Taylor homogenizational approach (left) and combined elastic and chemical energy (right) for a linear (solid, dash-dot), a cubic (dashes, dash-dot-dot) and a scaled tangent hyperbolicus (small dashes, dots) interpolation function $h$

$$
h_{\tanh }(\phi)=\frac{1}{2} \tanh \left(\theta\left(\phi-\frac{1}{2}\right)\right)+\frac{1}{2},
$$

which are referred to as linear, cubic, or a tangent hyperbolicus scaling function, respectively. While the linear and cubic functions satisfy equation (33) perfectly, however, the tangent hyperbolicus function does not. If $\theta=14$ a residual error of $1-h_{\tanh }(\phi=1)=h_{\tanh }(\phi=0) \approx 10^{-6}$ remains [38].

\section{Voigt/Taylor approach}

In the Voigt/Taylor approach, the strain is assumed to be uniform in each phase, see, e.g., [5,23,37,42], such that

$$
\underset{\sim}{\varepsilon}={\underset{\sim}{1}}_{1}=\ldots={\underset{\sim}{\alpha}}_{\alpha}=\ldots={\underset{\sim}{n}}_{n} .
$$

The stress in a phase $\alpha$ is defined as the derivative of the energy with respect to the elastic strain

$$
\underset{\sim \alpha}{\sigma}=\frac{\partial f_{\alpha}}{\partial \mathcal{\varepsilon}_{\alpha}^{e}}=\underset{\sim \alpha}{\mathbf{C}}: \varepsilon_{\alpha}^{e}
$$

and the stress in the interface region is interpolated with a scalar function $h$ as in (33)

$$
\underset{\sim}{\sigma}=\sigma_{\sim_{0}} h^{0}\left(\phi_{1}, \ldots, \phi_{n}\right)+\sum_{\alpha=1}^{n}{\underset{\sim \alpha}{\sigma}}_{\alpha} h\left(\phi_{\alpha}\right) .
$$

The local elastic energy is therefore

$$
f_{\mathrm{u}}=f_{\mathrm{u}_{0}} h^{0}\left(\phi_{1}, \ldots, \phi_{n}\right)+\sum_{\alpha=1}^{n} f_{\mathrm{u}_{\alpha}} h\left(\phi_{\alpha}\right) .
$$

A closer look at (41) and (40) reveals that the mechanical behavior within the phases can be chosen independently of how the phase field parameter evolves. Therefore, the derivative of the elastic energy with respect to the elastic strain remains the sum of all the derivatives within the phases. The same applies for the derivatives of the stress with respect to the strain. Until here, no assumption about the material behavior within the phase has been made, e.g., we only require the derivative of the stress w.r.t. strain in (40). Following (5), the constitutive law in (39) within the individual phase $\alpha$ is formulated with

$$
\varepsilon_{\alpha}^{\varepsilon}={\underset{\sim}{\varepsilon}}_{\alpha}^{\mathrm{e}}+{\underset{\sim}{\varepsilon}}_{\alpha}^{*} .
$$


Fig. 2 (left) depicts the elastic energy for a two phase system $(\alpha=0,1)$, where the order parameter interpolates between the two phases. The nondimensionalized Young's moduli are $E_{0}=1$ and $E_{1}=1.1$ for the parent and the transformed phase, respectively; the Poisson's ratio is held constant $v=0.25$. The uniform strain and the transformation strain are given by

$$
\varepsilon_{\alpha}=\left[\begin{array}{cc}
0.09 & 0 \\
0 & 0
\end{array}\right] \text { and } \varepsilon_{\alpha}^{*}=\left[\begin{array}{cc}
0.02 & 0 \\
0 & 0.05
\end{array}\right] .
$$

The parameters are chosen in order to discuss the difference in the elastic energy for the Voigt/Taylor and the Khachaturyan approach. The energy behaves as described in (41) for the Voigt/Taylor case. Using the linear interpolation function $h_{\text {lin }}$, however, the second-order derivative with respect to the order parameter $\phi$ vanishes. That is not the case for the Khachaturyan approach, as both the effective material parameters, and the transformational strain are interpolated at least linearly. The downside is, however, that this results in an unphysical dip of the elastic energy across the interface due to their multiplication (Fig. 2), and effectively results in a stiffer interface than the bulk phases.

Fig. 2 (right) shows the combined elastic and chemical energy $f=f_{\mathrm{u}}+f_{\text {ch }}$ for a two phase system $(\alpha=0,1)$. Here, the proportional constant $\beta_{\mathrm{ch}}$ and the parameters of the Landau polynomial are given by

$$
\beta_{\mathrm{ch}}=5 \cdot 10^{-3}, \quad A=10, \quad B=42, \quad C=32, \quad \text { and } \quad D=1,
$$

such that the elastic $f_{\mathrm{u}}$ and the chemical energy $f_{\text {ch }}$ are in the same order of magnitude. Here, the global minimum for the Khachaturyan approach and the Voigt/Taylor approach with a linear interpolation function is not at $\phi=1$. It means that the minimization of this energy can lead to a solution, which does not represent a valid bulk phase value for $\phi$, and can even lie outside its valid range [0, 1].

\section{Implementation}

The Voigt/Taylor approach is implemented in the Finite Element Analysis Program FEAP [54] and in the Nonlinear Material and Structure Analysis Suite Z-set [55]. The degrees of freedom are $\underline{d}=\left(\underline{\phi}^{\top}, \underline{u}^{\top}\right)^{\top}$, where $\underline{u} \in \mathbb{R}^{d}$ and $\phi \in \mathbb{R}^{n}$. Here, $d=2,3$ is the number of spatial dimensions, and $n$ is the number of different ways to transform, where the components $\phi_{\alpha}$ of $\phi$ are associated with the corresponding transformational strain $\varepsilon_{\alpha}^{*}$. The number of phases $n$ and the transformational strain are dependent on the orientation relationship (OR). Common OR has been identified for the case of transforming face-centered cubic to body-centered cubic crystalline structures. The interested reader is referred to $[20,25]$ for a comprehensive overview. There are at least five orientation relationships, with the Bain OR [10] having the fewest transformation variants; however, more complicated cases such as the Nishiyama-Wassermann [39,58], Kurdjumov-Sachs [30], GreningerTroiano [18], or the Headley-Brooks OR [20] have been identified.

Using the Clausius-Duhem inequality in (7), the field equations are the balance of linear and angular momentum, and the evolution of the order parameters with a Ginzburg-Landau time-dependent evolution equation:

$$
\begin{gathered}
\underline{\nabla} \cdot \sigma=0 \\
\frac{\dot{\phi_{\alpha}}}{M}+\frac{\partial f_{\mathrm{u}}}{\partial \phi_{\alpha}}+\frac{\partial f_{\mathrm{ch}}}{\partial \phi_{\alpha}}-\left(\frac{\partial f_{\mathrm{grad}}}{\partial\left(\phi_{\alpha, i}\right)}\right)_{, i}=\pi_{\alpha}-\xi_{\alpha i, i}=0 .
\end{gathered}
$$

In view of the flux-divergence theorem, Green's identity, the weak form of the mechanical problem and the weak form of the evolution equation are obtained, which are useful for FE implementations, given by

$$
\begin{gathered}
\int_{V}\left(\underline{\nabla} \otimes \underline{\eta}_{\underline{u}}\right): \underset{\sim}{\sigma} \mathrm{d} V=\int_{\partial V} \underline{\eta}_{\underline{u}} \cdot(\underline{\sigma} \underline{n}) \mathrm{d} S \\
\int_{V} \frac{\dot{\phi_{\alpha}}}{M} \eta_{\phi_{\alpha}}+\frac{\partial f_{\mathrm{u}}}{\partial \phi_{\alpha}} \eta_{\phi_{\alpha}}+\frac{\partial f_{\mathrm{ch}}}{\partial \phi_{\alpha}} \eta_{\phi_{\alpha}}+\alpha\left(\underline{\nabla} \phi_{\alpha}\right) \cdot\left(\underline{\nabla} \eta_{\phi_{\alpha}}\right) \mathrm{d} V \\
=\int_{\partial V} \eta_{\phi_{\alpha}}\left(\underline{\nabla} \phi_{\alpha} \cdot \underline{n}\right) \mathrm{d} S,
\end{gathered}
$$


where $\underline{n}$ is the outer normal vector and $\underline{\eta}_{u}$ and $\eta_{\phi_{\alpha}}$ are the test functions for the mechanical problem and the phase $\phi_{\alpha}$. The values $\underline{u}$ and $\phi_{\alpha}$ are discretized with ansatz functions $N_{I} \in \mathbb{R}$, where $I$ represents the index of the node. Nodal values are superscript with a hat (.). Discretized vectors and scalar quantities $a$, e.g., $\underline{u}, \underline{\phi}, \phi_{\alpha}$, $\dot{\phi}, \dot{\phi}_{\alpha}$, are denoted with a superscript (.) $)^{h}$, reading

$$
a^{h}=\sum_{I=1}^{N} N_{I} \hat{a}_{I} .
$$

The discretizations for the strain tensor and the gradient of the components of the order parameter $\phi_{\alpha}$ are

$$
\begin{aligned}
& \underline{\varepsilon}^{\mathrm{h}}=\sum_{I=1}^{N} \underline{\sim}_{I}^{u} \cdot \underline{\hat{u}}_{I} \quad \text { and } \quad \underline{\nabla} \phi_{\alpha}^{\mathrm{h}}=\sum_{I=1}^{N} \underline{B}_{I}^{\phi} \hat{\phi}_{\alpha I} \text {, where } \\
& \underline{\mathbf{B}}_{I}^{u}=\left[\begin{array}{ccc}
N_{, 1} & 0 & 0 \\
0 & N_{, 2} & 0 \\
0 & 0 & N_{, 3} \\
0 & N_{, 3} & N_{, 2} \\
N_{, 3} & 0 & N_{, 1} \\
N_{, 2} & N_{, 1} & 0
\end{array}\right] \quad \text { and } \quad \underline{B}_{I}^{\phi}=\underline{\nabla} N_{I} \in \mathbb{R}^{3}
\end{aligned}
$$

7.1 Residual and elemental stiffness matrix

Using the weak forms, the residual vector $\underline{R}_{I} \in \mathbb{R}^{(d+n)}$ of the node $I$ can be described with the nodal degrees of freedom $\underline{\hat{d}}_{J}=\left(\underline{\hat{u}}_{J}^{\top}, \underline{\hat{\phi}}_{J}^{\top}\right)^{\top}$ and the time derivatives $\underline{\hat{\dot{d}}}_{J}=\underline{\hat{\dot{\phi}}}_{J}$ on a node $J$

$$
\begin{gathered}
\underline{R}_{I}\left(\underline{\hat{d}}_{J}, \underline{\hat{\dot{d}}}_{J}\right)=\left(\begin{array}{l}
\underline{R}_{I}^{\underline{u}}\left(\underline{\hat{d}}_{J}\right) \\
\underline{R}_{I}^{\underline{\phi}}\left(\underline{\hat{d}}_{J}, \underline{\hat{\dot{d}}}_{J}\right)
\end{array}\right), \quad \underline{R}_{I}^{u} \in \mathbb{R}^{d} \quad \text { and } \underline{R}_{I}^{\phi}\left(\underline{\hat{d}}_{J}, \underline{\hat{\dot{d}}}_{J}\right)=\sum_{\alpha=1}^{n} R_{I}^{\phi_{\alpha}}\left(\underline{\hat{d}}_{J}, \underline{\hat{d}}_{J}\right) \underline{e}_{\alpha} \in \mathbb{R}^{n}, \\
\underline{R}_{I}^{u}=\int_{V}\left(\underline{\mathbf{B}}_{I}^{u}\right)^{\top} \underline{\sigma} \mathrm{d} V, \\
\underline{R}_{I}^{\phi_{\alpha}}=\int_{V} \frac{\dot{\phi}_{i}}{M} N_{I}+\frac{\partial f_{\mathrm{u}}}{\partial \phi_{\alpha}} N_{I}+\frac{\partial f_{\mathrm{ch}}}{\partial \phi_{\alpha}} N_{I}+\alpha_{\mathrm{grad}}\left(\underline{\nabla} \phi_{\alpha}\right) \cdot \underline{B}_{I}^{\phi} \mathrm{d} V .
\end{gathered}
$$

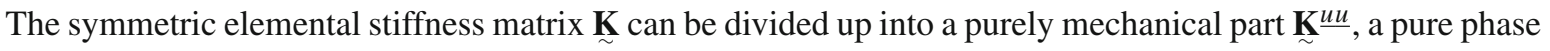

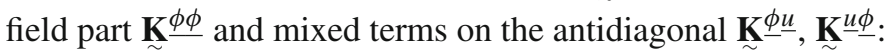

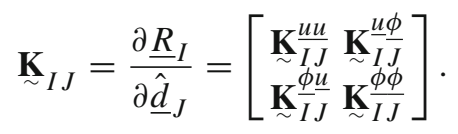

The matrix entries are

$$
\begin{aligned}
& \underset{\sim}{\mathbf{K}_{I J}^{u u}}=\frac{\partial \underline{R}_{I}^{u}}{\partial \underline{\hat{u}}_{J}}=\frac{\partial \underline{R}_{I}^{\underline{u}}}{\partial \underline{\varepsilon}^{\prime}} \frac{\partial \underline{\varepsilon}}{\partial \underline{\hat{u}}_{J}}=\int_{V}\left(\mathbf{B}_{I}^{\underline{u}}\right)^{\top} \frac{\partial \underline{\sigma}}{\partial \underline{\varepsilon}}\left(\underline{\mathbf{B}}_{J}^{\frac{u}{J}}\right) \mathrm{d} V,
\end{aligned}
$$

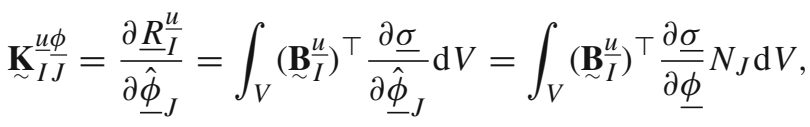




$$
\begin{aligned}
& K_{I J}^{\phi_{\alpha} \phi_{\beta}}=\frac{\partial \underline{R}_{I}^{\phi_{\alpha}}}{\partial \hat{\phi}_{\beta J}}=\int_{V} \frac{\partial^{2}\left(f_{\mathrm{ch}}+f_{\mathrm{u}}\right)}{\partial \phi_{\alpha} \partial \phi_{\beta}} N_{I} N_{J}+\alpha_{\operatorname{grad}} \underline{B}_{I}^{\phi} \frac{\partial\left(\phi_{\alpha, i}\right)}{\partial\left(\phi_{\beta, i}\right)} \frac{\partial\left(\phi_{\beta, j}\right)}{\partial \hat{\phi}_{j J}} \mathrm{~d} V \\
& =\int_{V} \frac{\partial^{2}\left(f_{\text {ch }}+f_{\mathrm{u}}\right)}{\partial \phi_{\alpha} \partial \phi_{\beta}} N_{I} N_{J}+\alpha_{\text {grad }} \underline{B}_{I}^{\phi} \delta_{\alpha \beta} \underline{B}_{J}^{\phi} \mathrm{d} V, \\
& \underline{K}_{I J}^{\phi_{\alpha} u}=\frac{\partial \underline{R}_{I}^{\phi_{\alpha}}}{\partial \underline{\hat{\hat{u}}}_{J}}=\int_{V} \frac{\partial^{2}\left(f_{\mathrm{ch}}+f_{\mathrm{u}}\right)}{\partial \phi_{\alpha} \partial \underline{u}} N_{I} \mathrm{~d} V \\
& =\int_{V} \partial\left(\frac{\partial f_{\mathrm{u}}}{\partial \underline{\varepsilon}^{\underline{\varepsilon}}} \frac{\partial \underline{\varepsilon}}{\partial \underline{\hat{u}}_{J}}\right) / \partial \phi_{\alpha} N_{I} \mathrm{~d} V \\
& =\int_{V} \partial\left(\underline{\sigma}^{\top} \underline{\mathbf{B}}_{\sim}^{\underline{u}}\right) / \partial \phi_{\alpha} N_{I} \mathrm{~d} V \text { with } \frac{\partial \mathbf{B}_{J}^{\underline{u}}}{\partial \phi_{\alpha}}=0 \\
& =\int_{V} N_{I}\left(\frac{\partial \underline{\sigma}}{\partial \phi_{\alpha}}\right)^{\top} \quad \mathbf{B}_{\sim}^{u} \mathrm{~d} V, \quad \text { and } \\
& \underset{\sim}{\mathbf{K}} \frac{\phi \underline{u}}{I J}=\left(\underset{\sim}{\underline{\underline{u}}} \frac{\underline{\phi}}{J I}\right)^{\top}=\int_{V} N_{I}\left(\frac{\partial \underline{\sigma}}{\partial \underline{\phi}}\right)^{\top} \underset{\sim}{\mathbf{B}} \frac{u}{J} \mathrm{~d} V .
\end{aligned}
$$

The damping matrix is dependent on the phase field terms only. It is given by

$$
\underset{\sim}{\mathbf{D}_{I J}}=\frac{\partial \underline{R}_{I}}{\partial \underline{\dot{d}}_{J}}=\left[\begin{array}{cc}
\underset{\sim}{\mathbf{0}} & \underset{\widetilde{\phi} \phi}{\mathbf{0}} \\
\underset{\sim}{\mathbf{D}} & \frac{\sim}{I J}
\end{array}\right],
$$

where the entries are

$$
D_{I J}^{\phi_{\alpha} \phi_{\beta}}=\delta_{\alpha \beta} \int_{V} \frac{N_{I} N_{J}}{M} \mathrm{~d} V
$$

7.2 Derivatives of the chemical potential

The chemical free energy expressed in (11) is not dependent on the strain; however, the following first- and second-order (mixed) derivatives w.r.t the order parameter $\phi_{\alpha}$ arise:

$$
\begin{aligned}
\frac{\partial f_{\mathrm{ch}}(\underline{\phi})}{\partial \phi_{\alpha}} & =A \phi_{\alpha}-B \phi_{\alpha}^{2}+C\left(\phi_{\beta} \phi_{\beta}\right) \phi_{\alpha} \\
\frac{\partial f_{\mathrm{ch}}(\underline{\phi})}{\partial \phi_{\alpha}} \phi_{\beta} & = \begin{cases}A-2 B \phi_{\alpha}+2 C \phi_{\alpha} \phi_{\beta}-C \phi_{\gamma} \phi_{\gamma}, & \text { where } \alpha=\beta \\
2 C \phi_{\alpha} \phi_{\beta}, & \text { where } \alpha \neq \beta\end{cases}
\end{aligned}
$$

Subsequently, the first and second derivatives of elastic energy w.r.t. the elastic strain for the mechanical problem $\left(\frac{\partial \sigma}{\partial \mathcal{E}^{\mathrm{e}}}, \sigma=\frac{\partial f_{\mathrm{u}}}{\partial \mathcal{E}^{\mathrm{e}}}\right)$ are given in the constitutive material law. The elastic energy within the interface is given by (41). The derivatives w.r.t to the order parameter are

$$
\begin{aligned}
\frac{\partial f_{\mathrm{u}}}{\partial \phi_{\alpha}}(\underline{\phi}, \underline{u}) & =h^{\prime}\left(\phi_{\alpha}\right)\left(f_{\mathrm{u} \alpha}-f_{\mathrm{u} 0}\right) \\
\frac{\partial f_{\mathrm{u}}}{\partial \phi_{\alpha}} \phi_{\beta}(\underline{\phi}, \underline{u}) & =\left\{\begin{array}{ll}
h^{\prime \prime}\left(\phi_{\alpha}\right)\left(f_{\mathrm{u} \alpha}-f_{\mathrm{u} 0}\right), & \text { where } \alpha=\beta \\
0, & \text { where } \alpha \neq \beta
\end{array} .\right.
\end{aligned}
$$

and depend on the choice of $h$ (see equations $35,36,37$ ) only. Their derivatives are given by

$$
h_{\text {linear }}(\phi)^{\prime}=1, \quad h_{\text {linear }}(\phi)^{\prime \prime}=0,
$$


Table 1 Overview of different phases and interpolation methods. The x marks where a simulation was conducted here

\begin{tabular}{|c|c|c|c|c|}
\hline & \multicolumn{2}{|l|}{$n=2$} & \multicolumn{2}{|l|}{$n=3$} \\
\hline & $\overline{h_{\mathrm{an}}}$ & $h_{\text {if }}$ & $\overline{h_{\text {an }}}$ & $h_{\text {if }}$ \\
\hline$h_{\text {linear }}$ & $\mathrm{x},[45]$ & equivalent to $h_{\mathrm{an}}$ & $\mathrm{x},[46]$ & equivalent to $h_{\mathrm{an}}$ \\
\hline$h_{\text {cubic }}$ & $\mathrm{x}$ & $\mathrm{x}$ & wrong solution & $\mathrm{x}$ \\
\hline$h_{\tanh }$ & $\mathrm{x},[38]$ & $\mathrm{x}$ & no convergence & $\mathrm{x}$ \\
\hline
\end{tabular}

$$
\begin{array}{rlrl}
h_{\text {cubic }}(\phi)^{\prime} & =6 \phi-6 \phi^{2}, & & h_{\text {cubic }}(\phi)^{\prime \prime}=6-12 \phi, \\
h_{\tanh }(\phi)^{\prime} & =\frac{1}{2} \alpha\left(1-\tanh \left(\alpha\left(\phi-\frac{1}{2}\right)\right)^{2}\right), & \\
h_{\tanh }(\phi)^{\prime \prime} & =-2 \alpha \tanh \left(\alpha\left(\phi-\frac{1}{2}\right)\right) h_{\tanh }(\phi) . &
\end{array}
$$

In a preliminary test, the number of phases present $(n=2,3)$, the interpolation methods $\left(h_{\mathrm{an}}, h_{\mathrm{if}}\right)$ and the interpolation functions ( $h_{\text {linear }}, h_{\text {cubic }}, h_{\text {tanh }}$ ) have been varied. In the case of two phases, a two-phase beam splits vertically in two halves where the left half is initialized with parent phase $\left(\phi_{1}=0\right)$ and the right with the child phase $\left(\phi_{1}=1\right)$ is considered. In the case of three phases $(n=3)$, we consider a three-phase beam as depicted in Fig. 3 (a). Table 1 gives an overview of the interpolation methods for a varying number of phases. Where applicable, a literature reference is give. Cases successfully tested by us are marked with an $\mathrm{x}$; otherwise, a comment on the issue is provided. As shown in Sect. 3, if either considering two phases or a linear $h_{\text {linear }}$, the partition of unity (31) holds equally. However, when considering three different phases, while using the analogous approach $h_{\text {an }}$ for the interpolation, we do not necessarily obtain the correct solution or no solution at all. In the case of a cubic interpolation function $h_{\text {cubic }}$, the whole beam transforms to a homogeneous interface region. It is filled with a valid, however, unphysical solution. In the case of a tanh interpolation function $h_{\text {tanh }}$, no convergence and therefore no solution can be obtained.

\section{Verification}

A verification example is presented in Fig. 3. Consider a beam of 176nm length and 35.2nm height under load, realized as a displacement of the far ends of the beam. The initial configuration is shown in Fig. 3 (a), where the left quarter is initialized with the first martensitic orientation variant $\mathrm{M}_{1}$, the right quarter is initialized with the second martensitic orientation variant $\mathrm{M}_{2}$, and the center section of the beam is endowed with austenite. Before discussing the simulation, we make some basic consideration, regarding the chemical and elastic energy separately in a simplified setting.

We consider a temperature of $T=300 \mathrm{~K}$. Following [44], the parameters of the temperature-dependent parameters of the Landau polynomial read

$$
A=9.7187, \quad B=9.7187, \quad C=31.4375, \quad \text { and } \quad D=1 .
$$

With that, the martensitic phase is stable. Considering the chemical energy only, while disregarding the elastic energy, Austenite transforms into martensite. With the initial configuration given in Fig. 3 (a), both interfaces $M_{1}-A$ and $M_{2}-A$ would proceed at the same speed toward the center, transforming austenite into the first and second martensitic orientation variants. Eventually, they meet in the center, forming a single interface $\mathbf{M}_{2}-\mathbf{M}_{1}$. For the martensitic phases, an eigenstrain is introduced

$$
\underset{\sim 1}{\varepsilon_{1}^{*}}=\left[\begin{array}{cc}
0.05 & 0 \\
0 & 0
\end{array}\right] \quad \text { and } \quad \varepsilon_{2}^{*}=\left[\begin{array}{cc}
0 & 0 \\
0 & 0.05
\end{array}\right],
$$

such that the first orientation variant imposes a strain in $x_{1}$-direction and the second orientation variant in $x_{2}$ direction. Therefore, a strain or force in $x_{1}$-direction influences the elastic energy and therefore the formation of the first orientation variant more strongly compared to the formation of the second variant, which is only affected by the lateral strain. The same applies, for a strain or force in $x_{2}$-direction and the second orientation variant, however, interchanged.

Now, we disregard the chemical energy and neglect the lateral strain, while considering the elastic energy only. A displacement of the far ends of the beam is applied in $x_{1}$-direction. With that, the second martensitic 


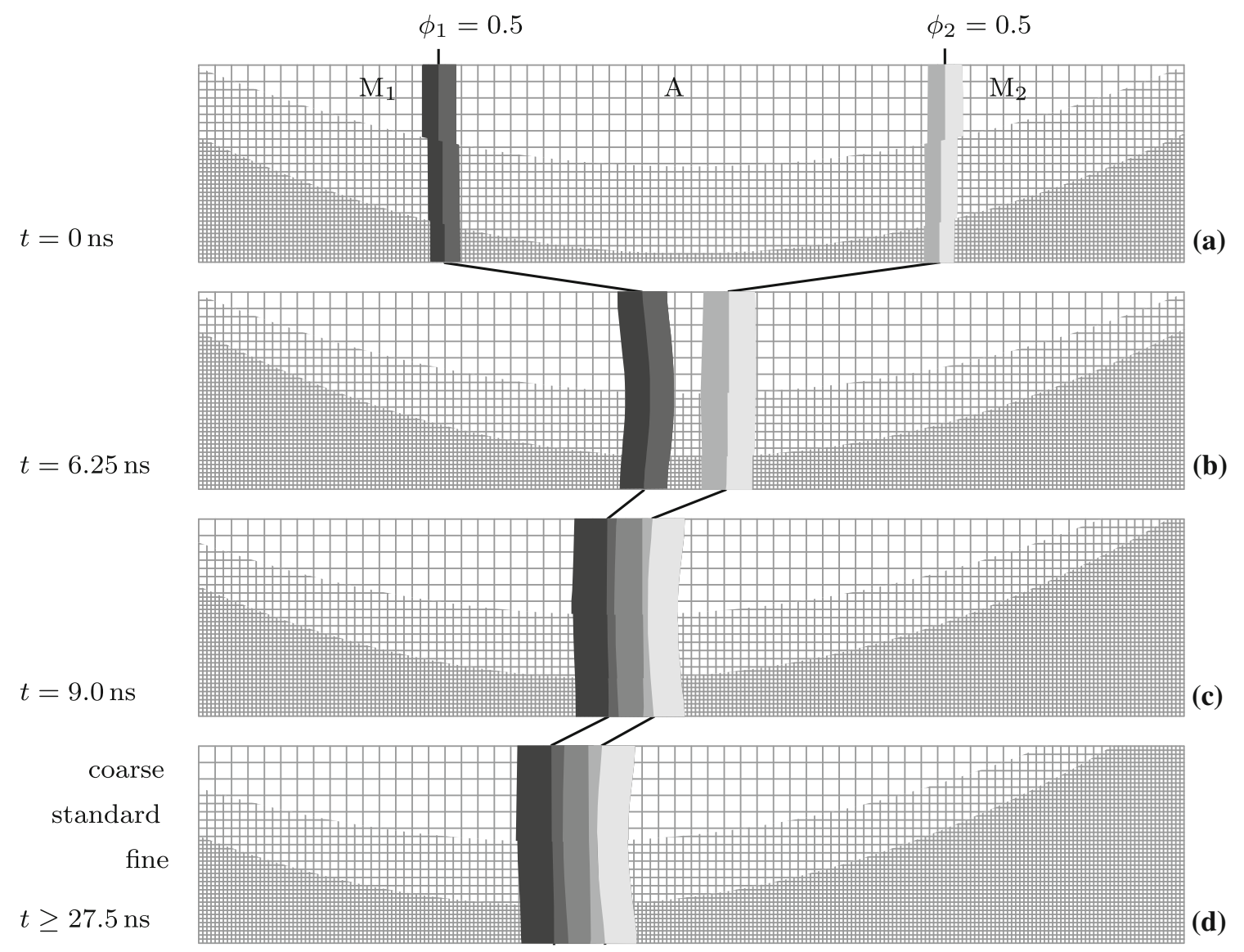

Fig. 3 Time evolution of the martensitic transformation for different levels of mesh refinement. The solid colored regions indicate the interface region where $0.2<\phi_{1}<0.8$ (left, dark grey), and where $0.2<\phi_{2}<0.8$ (right, light grey). Initially, the austenitic phase is present in the center $(A)$, with the martensitic orientation variants to the left $\left(\mathrm{M}_{1}\right)$ and right $\left(\mathrm{M}_{2}\right)$

orientation variant $M_{2}$ and the austenitic phase $A$ are energetically indistinguishable, as neither imposes an eigenstrain in $x_{1}$-direction. As a consequence, we disregard the presence of the martensitic orientation variant $M_{2}$ altogether and replace it with austenite. A displacement of $d=8.8 \mathrm{~nm}$ amounts to $5 \%$ total strain, accommodating the $\mathrm{M}_{1}$ phase perfectly. The final configuration in that case is a beam of pure $\mathrm{M}_{1}$. With the far ends clamped, where $d=0 \mathrm{~nm}$, the austenitic phase is stable. Any other displacement between 0 and $8.8 \mathrm{~nm}$ accommodates both phases $M_{1}$ and A partly. For example, a displacement of $d=4.4 \mathrm{~nm}$, amounting to $2.5 \%$ total strain, leads to a final configuration, where half of the beam is austenitic, and the other half is of pure martensite $\mathrm{M}_{1}$. In that case, the final configuration can be described with the interface position at $x_{1 \mathrm{M}_{1} \mathrm{~A}}=88 \mathrm{~nm}$. The interface position can be determined with a ratio

$$
x_{1 \mathrm{M}_{1} \mathrm{~A}}=\frac{d}{\left(\varepsilon_{1}^{*}\right)_{11}},
$$

where $\left(\varepsilon_{1}^{*}\right)_{11}$ is the 11-component of the transformation strain tensor $\varepsilon_{1}^{*}$. For example, a strain of $2 \%$, tantamount to $d=3.52 \mathrm{~nm}$, leads in this simplified case to an interface position of $x_{1 \mathrm{M}_{1} \mathrm{~A}}=70.4 \mathrm{~nm}$.

In the simulation, both the elastic and the chemical energies are considered with the parameters as mentioned above. The Voigt/Taylor approach with a cubic interpolation function is used. The elasticity matrices for pure iron in the austenitic and the martensitic phase are given by

$$
\underset{\approx 0}{\mathbf{C}}=\underset{\approx \mathrm{Fe}, \text { fcc, simpl. }}{\mathbf{C}}=\left[\begin{array}{ccc}
2.2 & 1.5 & 0 \\
1.5 & 2.2 & 0 \\
0 & 0 & 0.3
\end{array}\right] \cdot 10^{5} \mathrm{~N} \mathrm{~mm}^{-2} \text {, and }
$$


Table 2 Interface position in dependence of the mesh compared with the analytic solution

\begin{tabular}{llllll}
\hline mesh & $n_{\mathrm{el}}$ & $n_{\mathrm{el} x_{1}}$ & $n_{\mathrm{el} x_{2}}$ & $n_{\mathrm{el} x_{1}} \frac{L}{l}$ & $x_{1\left(\mathrm{M}_{1} \mathrm{~A}\right)}$ \\
\hline coarse & 720 & 60 & 12 & 10.23 & $67.48 \mathrm{~nm}$ \\
standard & 2880 & 120 & 24 & 20.45 & $67.54 \mathrm{~nm}$ \\
fine & 11520 & 240 & 48 & 40.91 & $67.55 \mathrm{~nm}$ \\
analytic & - & - & - & - & $70.40 \mathrm{~nm}$ \\
\hline
\end{tabular}

$$
\underset{\approx 1}{\mathbf{C}}=\underset{\approx 2}{\mathbf{C}}=\underset{\approx \mathrm{Fe}, \text { bcc, simpl. }}{\mathbf{C}}=\left[\begin{array}{ccc}
2.9 & 1.6 & 0 \\
1.6 & 2.9 & 0 \\
0 & 0 & 0.6
\end{array}\right] \cdot 10^{5} \mathrm{~N} \mathrm{~mm}^{-2} .
$$

Please note that in contrast to [46] the parameters have been simplified. However, they are in the right order of magnitude. The parameters for the chemical energy and the mobility are

$$
G=0.96 \cdot 10^{-6} \frac{\mathrm{J}}{\mathrm{mm}}, \quad L=30 \mathrm{~nm}, \text { and } \quad M=9.6 \cdot 10^{-3} \frac{\mathrm{mm}}{\mathrm{N} \mathrm{ns}} .
$$

In Fig. 3, the time evolution of the martensitic transformation is shown, overlapping the solutions for a coarse, a standard, and a fine mesh. The initial configuration in Fig. 3 (a) is followed by intermediate steps (b, c), leading to the final configuration (d). First, a transformation from the martensitic to the austenitic phase takes place. That means that both martensitic variants are energetically more stable than the austenitic phase. This can be explained illustratively: Both the first and the second martensitic orientation variants are chemically more stable than the austenitic phase. As the strain of the beam is in $x_{1}$-direction, the transformation strain entry $\left(\varepsilon_{\alpha}^{*}\right)_{11}$ is of importance. In terms of the elastic energy, the second orientation variant and the austenitic phase are almost equal, as the transformation strain entries vanish $\left(\left(\varepsilon_{0}^{*}\right)_{11}=\left(\varepsilon_{1}^{*}\right)_{11}=0\right)$. However, due to the chemical energy, the second martensitic orientation variant is preferred to the austenite. The interface motion between the time frames is indicated schematically by the lines connecting the contours of constant $\phi_{1}=0.5$, and $\phi_{2}=0.5$.

The velocity of the $\mathrm{M}_{2} \mathrm{~A}$ interface is marginally higher than the velocity of the $\mathrm{M}_{1} \mathrm{~A}$ interface. That is because the second martensitic orientation variant is, in terms of the elastic energy, slightly more favorable than the first martensitic orientation variant. The phases move toward each other as depicted in Fig. 3 (b). Eventually, they join, forming a mutual interface. Thereafter, they proceed to move toward the left as shown in Fig. 3 (c). As both martensitic phases are chemically equally stable, equation (72) provides a good approximation for the final configuration.

Considering the elastic energy only, with no lateral strain and without varying elasticity matrices, this leads to an interface position of $70.4 \mathrm{~nm}$. The interface position in the simulations can be calculated by averaging the $x_{1}$-position where $\phi_{1}=\phi_{2}$ over the $x_{2}$-coordinate. The simulation has been conducted with three differently refined meshes, termed coarse, standard, and fine. The standard mesh consists of 2880 elements, while the coarse mesh is four times coarser amounting to 720 elements. The fine mesh is at 11520 elements. The positions for the interface $\mathrm{M}_{1} \mathrm{M}_{2}$ are at $67.48 \mathrm{~nm}$ for the coarse mesh, $67.54 \mathrm{~nm}$ for the standard refined mesh, and $67.55 \mathrm{~nm}$ for the fine mesh. The digits below the atom width illustrate that the results obtained from all mesh refinements are physically equivalent. Furthermore, they are well in line with the prediction in (72). The results are summed up in table 2, where in addition to the number of elements in $x_{1}$ and in $x_{2}$ direction, $n_{\mathrm{el} x_{1}}$, and $n_{\mathrm{el}_{x_{2}}}$, respectively, the mesh density as elements per specific interface width $L$ is given $\left(n_{\mathrm{el}_{x_{1}}} \frac{L}{l}\right)$. The coarse mesh has about ten elements per interface width $L$, where the values for the standard and the fine mesh are twofold and fourfold higher. As a result, the coarse mesh does not considerably vary from the fine mesh, even less so the result obtained using the standard mesh. Therefore, a number of ten elements per interface width $L$ are sufficient.

\section{Comparison of the Voigt/Taylor with the Khachaturyan approach}

In the following, we compare the Voigt/Taylor approach with a cubic interpolation function to the Khachaturyan approach presented in [46] with a system of three phases. In the Khachaturyan case, a linear interpolation 

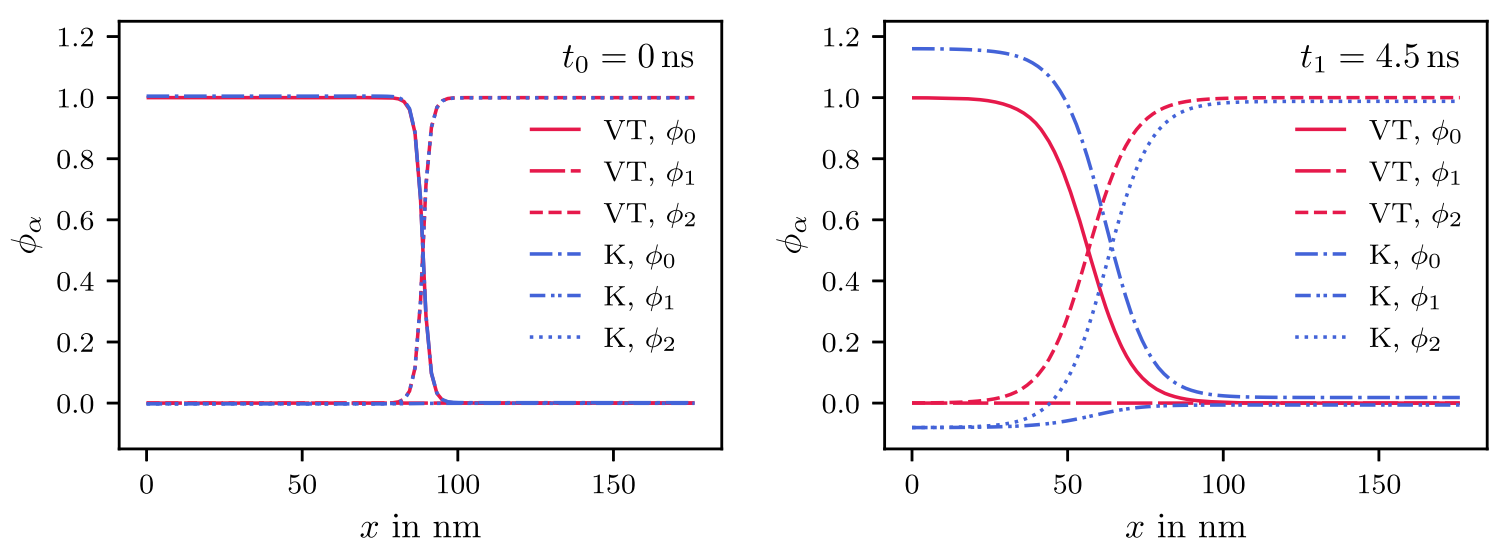

Fig. 4 Phase field for the parent phase $\phi_{0}$ (solid, dash-dot), and the martensitic orientation variants $\phi_{1}$ (dashes, dash-dot-dot) and $\phi_{2}$ (small dashes, dots) for the Khachaturyan using a linear interpolation (K, blue) and the Voigt/Taylor approach using a cubic interpolation (VT, red). The solution at $t_{0}$ is shown on the left, the evolution after $t_{1}=4.5 \mathrm{~ns}$ on the right (color figure online)
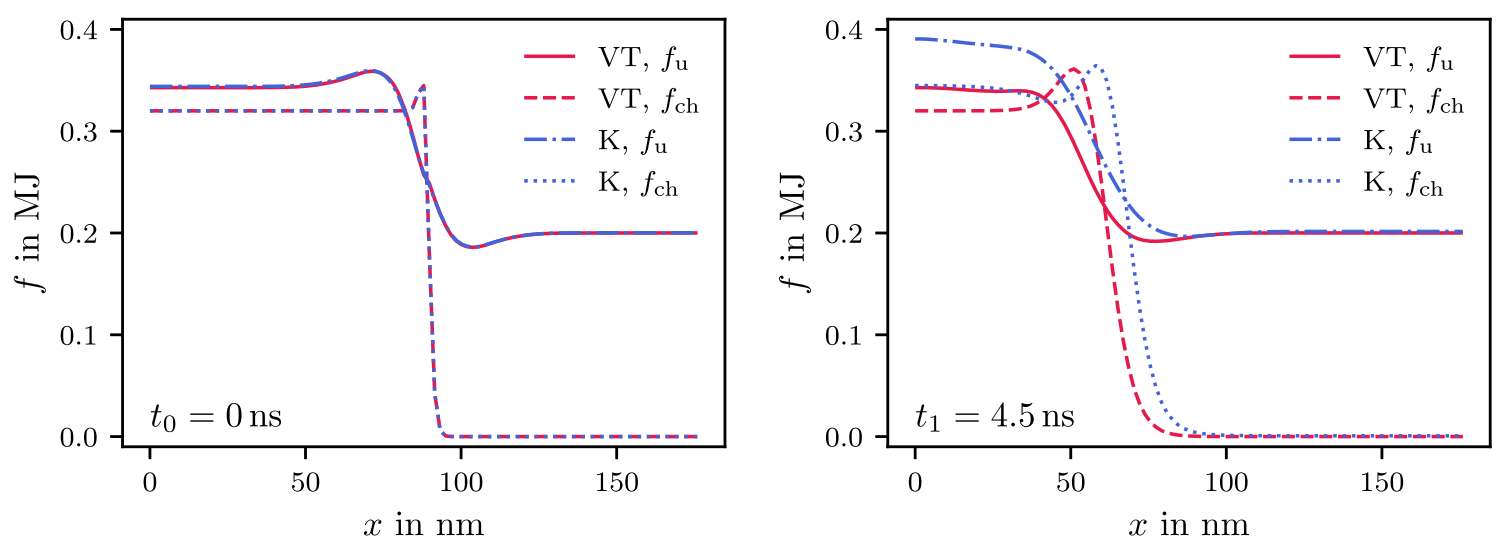

Fig. 5 Elastic energy $f_{\mathrm{u}}$ (solid, dash-dot) and chemical energy $f_{\text {ch }}$ (dashes, dots) for the Khachaturyan using a linear interpolation $\left(\mathrm{K}\right.$, blue) and the Voigt/Taylor approach using a cubic interpolation (VT, red). The solution at $t_{0}$ is shown on the left, the evolution after $t_{1}=4.5 \mathrm{~ns}$ on the right (color figure online)

function is used. The material moduli matrix for the austenitic $\mathbf{C}_{\mathrm{A}}$ and the martensitic $\mathbf{C}_{\mathrm{M}}$ phases are given by

$$
\mathbf{C}_{\mathrm{A}}=\left[\begin{array}{ccc}
2.2 & 1.5 & 0 \\
1.5 & 2.2 & 0 \\
0 & 0 & 0.3
\end{array}\right] 10^{5} \mathrm{~N} \mathrm{~mm}^{-2} \text { and } \mathbf{C}_{\mathrm{M}}=\left[\begin{array}{ccc}
2.9 & 1.6 & 0 \\
1.6 & 2.9 & 0 \\
0 & 0 & 0.4
\end{array}\right] 10^{5} \mathrm{~N} \mathrm{~mm}^{-2}
$$

The mobility constant is $M=9.6 \cdot 10^{6} \mathrm{~N} \mathrm{~mm}^{-2}$, the length scale $L=3 \mathrm{~nm}$, and the parameter influencing the characteristic energy density is $G=0.96 \mathrm{~J} \mathrm{~m}^{-1}[46,57]$. The temperature is held constant at $T=300 \mathrm{~K}$. In order to discuss the influence of the change in the elastic constant across the phases, the eigenstrain is neglected. This is, for example, true in the habit plane with invariant plane strain [28]. For the geometry, a beam of $a=176 \mathrm{~nm}$ length and $b=\frac{1}{5} a$ width is chosen. The initial beam is initialized with no presence of the first martensitic orientation variant $\phi_{1}=0$, where $\phi_{0}=1$ in the left half of the beam, and $\phi_{2}=1$ in the right half of the beam. A distributed load of $F=10^{-3} \mathrm{~N}$ is applied to the far ends of the beam, leading to a total strain within the order of magnitude of $3 \%$.

Fig. 4 shows the local order parameter field at time $t_{0}=0 \mathrm{~ns}$ and after evolution at time $t_{1}=4.5 \mathrm{~ns}$. The distribution of the elastic and the chemical energy is shown in Fig. 5, again at time $t_{0}$ and after evolution at time $t_{1}$. Both the order parameter field, the elastic, and the chemical energy are equal in either of the two (Khachaturyan and Voigt/Taylor) cases. The elastic energy $f_{u}$ reaches the elastic energy $f_{0}$ and $f_{2}$ in the bulk phases given the local strain distribution. The chemical energy vanishes in the bulk child phases, where $\phi_{0}=0$ due to the nature of the Landau polynomial in equation (Eq. 11). In the austenitic phase $\left(\phi_{0}=0\right)$ it reaches a value of $\frac{G}{L}=0.32 \mathrm{MJ} \mathrm{m}^{-2}$. Both models rely on energy minimization. A transformation from any bulk phase 

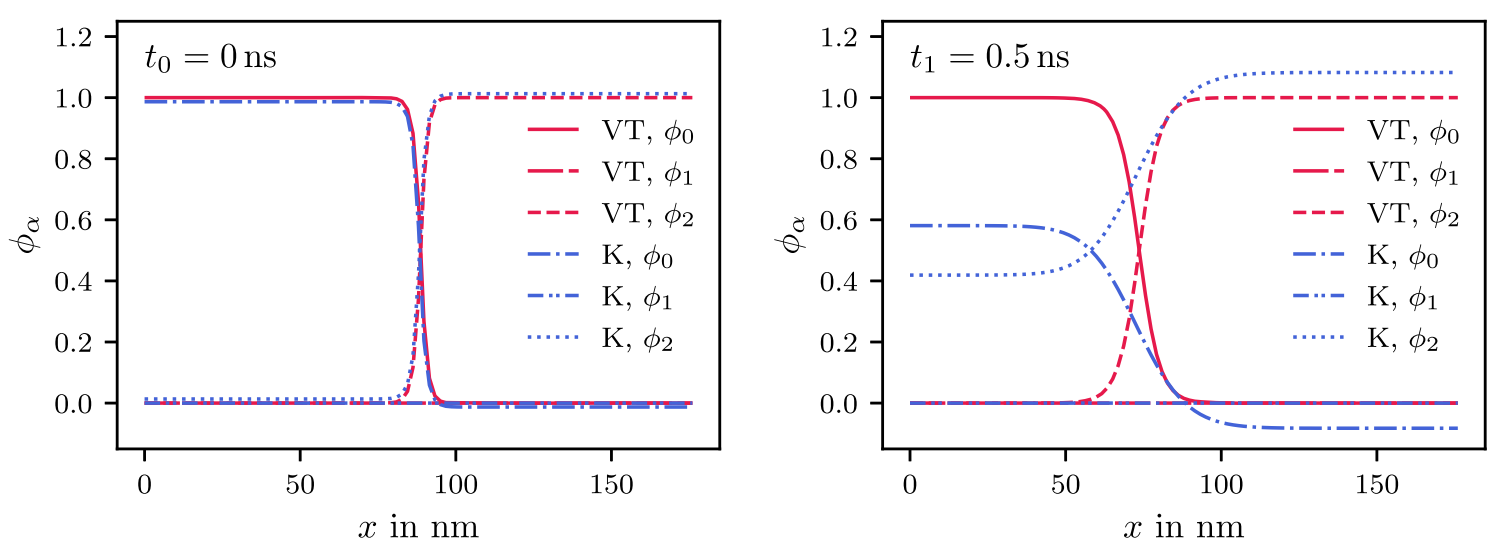

Fig. 6 Phase field for the parent phase $\phi_{0}$ (solid, dash-dot), and the martensitic orientation variants $\phi_{1}$ (dashes, dash-dot-dot) and $\phi_{2}$ (small dashes, dots) for the Khachaturyan using a linear interpolation (K, blue) and the Voigt/Taylor approach using a cubic interpolation (VT, red). The solution at $t_{0}$ is shown on the left, and the evolution after $t_{1}=0.5 \mathrm{~ns}$ on the right

to any other bulk phase introduces an interface, which is widened due to the gradient term, or in other terms the gradient term is minimized by minimizing the gradient of the phase field, which is achieved by widening the interface. Once the interface is established, the energy contribution of the chemical energy wants to minimize the interface width, therefore counteracting the gradient term and thus stabilizing the interface. As the terms counteract themselves on the interface and vanish for the bulk, we disregard them momentarily. The elastic and the chemical energy in the bulk phases of the solution at $t_{0}$ are an indicator for the stable solution of this problem: The global minimum is a transformation from the austenitic to the martensitic variant. In the Voigt/Taylor approach, the interface widens, while transforming to the martensitic variant as the phase field in Fig. 4 indicates. At all times the energy for the bulk phase energies are assumed by the respective bulk phases. Using the Khachaturyan approach, however, the chemical and elastic energy exceed the values for the bulk phases. A comparison with the phase field in Fig. 4 at time $t_{1}=4.5 \mathrm{~ns}$ shows that the order parameter exceeds its valid range. This is due to the use of a linear interpolation function in the Khachaturyan model. The combined elastic and chemical energy in Fig. 2 (right) offer an explanation. Both energies are in the same order of magnitude, such that solutions of the phase field not representing a bulk value are possible. This issue can be fixed by either using a nonlinear interpolation function like the cubic, which ensures that exceeding the values for the bulk phase leads to a bigger contribution as staying in the interface or the bulk, by using a tanh type interpolation function, limiting the phase field or by using an obstacle potential as in [51].

As a second test, in order to discuss the influence of the transformation strain the material moduli are constant for all the phases

$$
\mathbf{C}_{\mathrm{A}}=\mathbf{C}_{\mathrm{M}}=\left[\begin{array}{ccc}
2.2 & 1.5 & 0 \\
1.5 & 2.2 & 0 \\
0 & 0 & 0.3
\end{array}\right] 10^{5} \mathrm{~N} \mathrm{~mm}^{-2},
$$

and a transformation strain of

$$
\varepsilon_{2}^{*}=\left[\begin{array}{cc}
0.05 & 0.0 \\
0.0 & 0.0
\end{array}\right]
$$

is introduced for the martensitic orientation variant $\left(\phi_{2}=1\right)$. A local total strain that is similar to the transformation strain causes the transformation to be energetically beneficial. This example uses the same geometry as in the example above, however, with no load but a strain of 0.05 applied to the far ends of the beam in $x$-direction. In a similar fashion, we discuss the phase field shown in Fig. 6 right after the static initialization at $t_{0}=0.0 \mathrm{~ns}$ and after evolution at a time $t_{1}=0.5 \mathrm{~ns}$. The solutions at $t_{0}$ of the Khachaturyan and the Voigt/Taylor approach differ slightly. This leads to the difference in the elastic energies. Contrary to what was shown for the uniform strain case in Fig. 2, the elastic energy of the Voigt/Taylor approach is exaggerated in the interface. That is due to the mismatch of the transformational strain and the interpolation of the elastic energy rather than interpolating the effective material parameters as in the Khachaturyan approach. This excess energy is unfavorable for numerical implementations, as the interface needs to resolved with a 

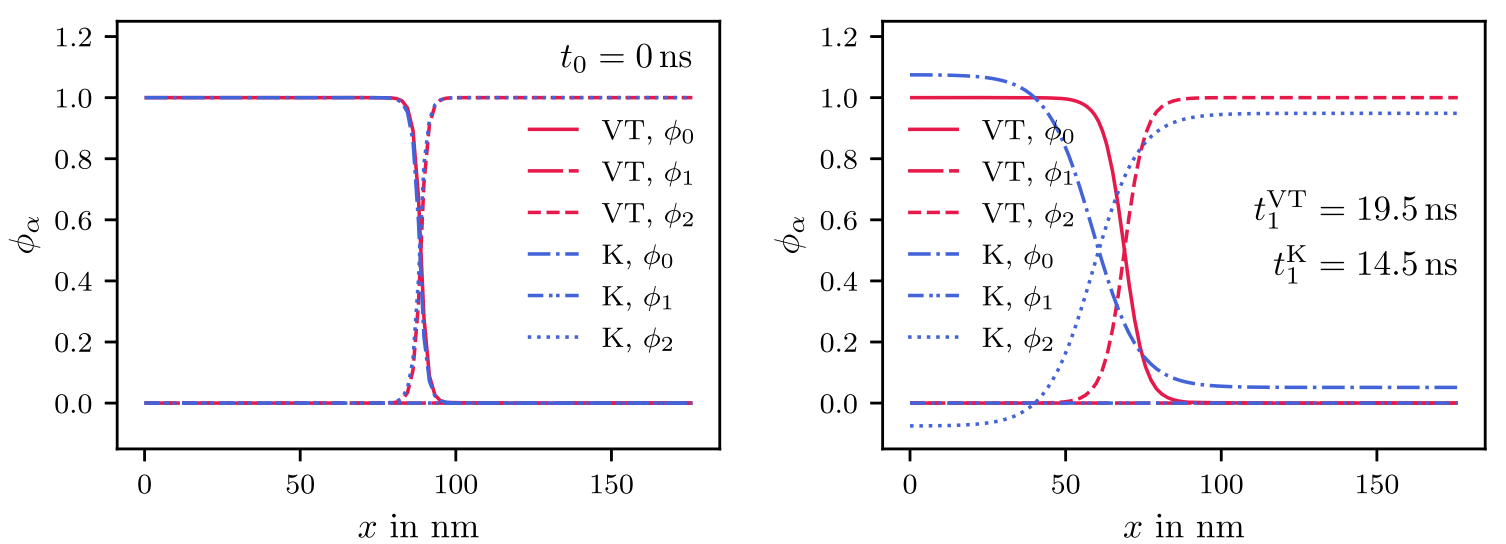

Fig. 7 Phase field for the parent phase $\phi_{0}$ (solid, dash-dot), and the martensitic orientation variants $\phi_{1}$ (dashes, dash-dot-dot) and $\phi_{2}$ (small dashes, dots) for the Khachaturyan using a linear interpolation (K, blue) and the Voigt/Taylor approach using a cubic interpolation (VT, red). The solution at $t_{0}$ is shown on the left, and the stable state after evolution is shown on the right (color figure online)

finer discretization. A countermeasure would be to raise the constant $L$ which is related to the interface width. Additionally, a different interpolation such as the ones proposed in $[11,48,49]$ could be used mitigating the excess energy within the diffuse interface. However, the Voigt/Taylor approach remains convincing, because the behavior of the phases can be chosen individually.

As the phases evolve, at the time of $t_{1}=0.5 \mathrm{~ns}$ an intermediate state is obtained. In the Khachaturyan approach, the interface is considerably wider. That is due to the lower and almost vanishing energy difference in the elastic energy. However, the order parameters $\phi_{0}$ and $\phi_{2}$ exceed their valid range. This is not the case for the Voigt/Taylor approach. Both phase field approaches end with the same solution $\phi_{2}=1$ across the whole domain.

The two test cases presented here, while behaving differently during the evolution, reach the same solution eventually. We modify the previously discussed test case to show that different solutions may be obtained. Disregarding the chemical energy contribution for the sake of the argument, a beam with no strain applied would favor the austenitic parent phase. On the other side, applying a strain of 0.05 , the martensitic phase would be obtained. A value in between should therefore favor a mix of the martensitic and the austenitic phase. Accordingly, the strain on the boundary in $x$-direction is reduced to 0.025 . Fig. 7 shows the solution of the phase field at $t_{0}$ and after converging to a stable solution. The solution for the Khachaturyan model is obtained for $t_{1}^{\mathrm{K}}=14.5 \mathrm{~ns}$. The bulk phases on the left side slightly exceed the valid range by approximately $6 \%$. The Voigt/Taylor approach is converged at $t_{1}^{\mathrm{VT}}=19.5 \mathrm{~ns}$. The position of the interface between the austenitic parent phase and the martensitic orientation variant is where $\phi_{0}=\phi_{2}=0.5$. The interface in the Khachaturyan case is at $x=60.7 \mathrm{~nm}$, and for the Voigt/Taylor case at $x=68.9 \mathrm{~nm}$.

In the previous cases, we compared the Khachaturyan approach with a scalar interpolation function to the Voigt/Taylor approach with a cubic interpolation function. This can lead to a wrong solution. The example presented in Fig. 8 shows the phase field at the initial and the stable state for the Khachaturyan and the Voigt/Taylor approach. Here, a cubic interpolation function is used in both cases. The final configuration in the Khachaturyan approach is reached at $8.25 \mathrm{~ns}$. The interface is located at $x_{1}=71.5 \mathrm{~nm}$, which is close to the interface in the Voigt/Taylor approach at $x_{1}=70.4 \mathrm{~nm}$.

\section{Conclusions}

We presented a comparison of the Voigt/Taylor approach and the Khachaturyan approach within a multivariant phase field for modeling the martensitic transformation. Here, both a chemical and a mechanical energy contribution are considered. The flowchart for the Voigt/Taylor approach is depicted in Fig. 9. The homogenization takes place after the material laws of the individual phases provide the elastic energy and their respective derivatives. Therefore, the material behavior of the parent and the child phases can be chosen independently. This stands in contrast to the Khachaturyan approach, where the effective material parameters are derived from interpolation to form a single material law. 

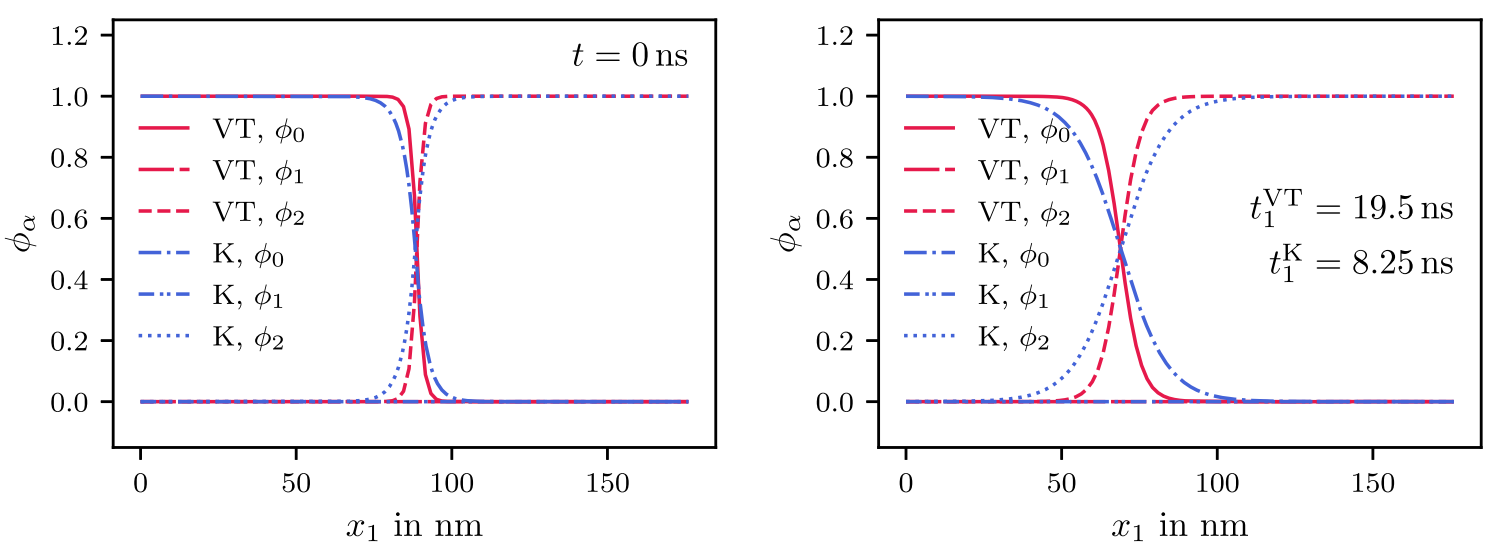

Fig. 8 Phase field for the parent phase $\phi_{0}$ (solid, dash-dot), and the martensitic orientation variants $\phi_{1}$ (dashes, dash-dot-dot) and $\phi_{2}$ (small dashes, dots) for the Khachaturyan using a linear interpolation (K, blue) and the Voigt/Taylor approach (VT, red). The solution at $t_{0}$ is shown on the left, and the stable state after evolution is shown on the right (color figure online)

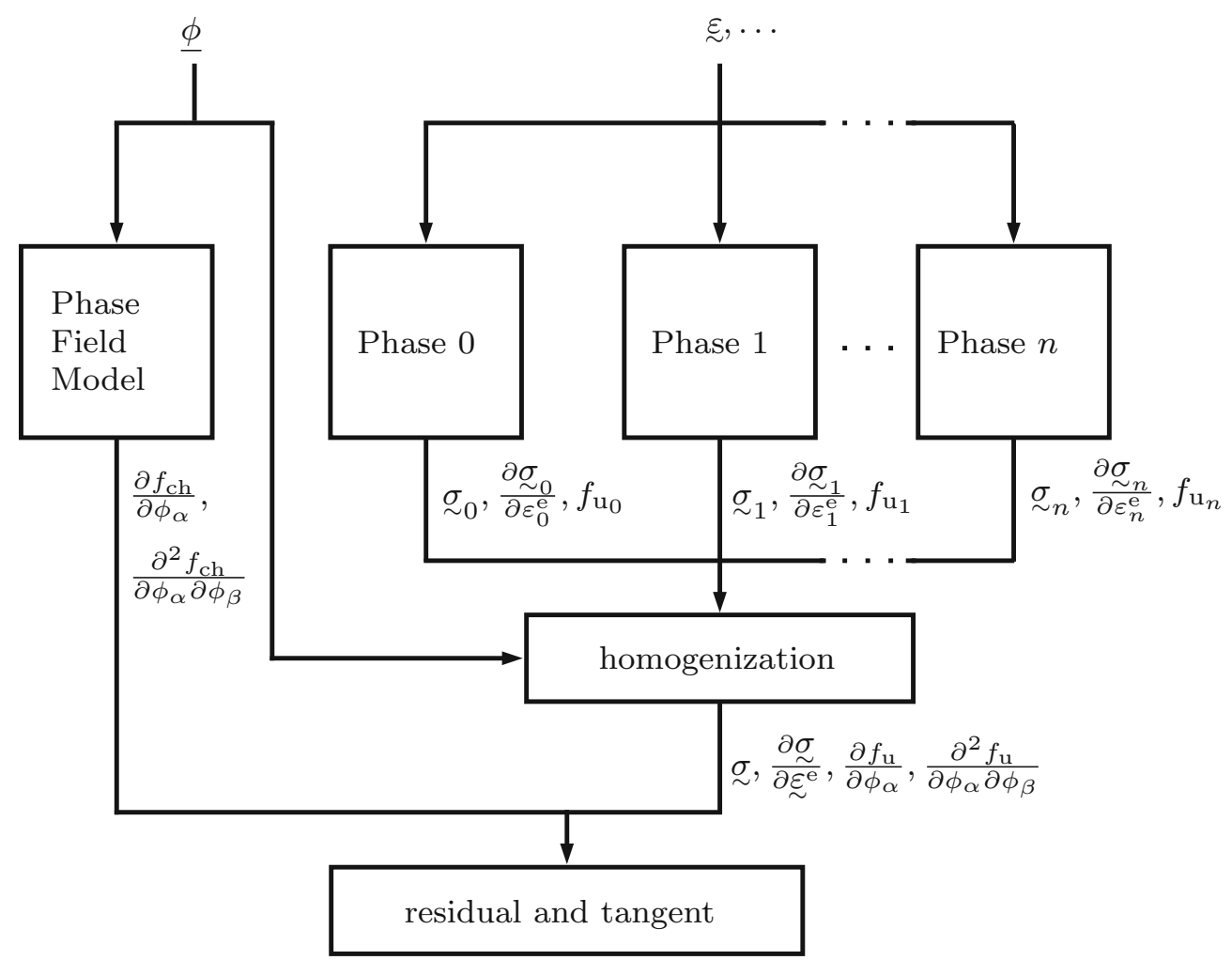

Fig. 9 Flowchart of the Voigt/Taylor homogenization approach for a phase field for the martensitic transformation adapted from [42]

Further, a potential drawback of the Khachaturyan approach is the unphysical drop in a uniform strain setting of the elastic energy, which is mitigated by the Voigt/Taylor scheme used here. However, as shown in the numerical examples, the mismatch of the austenitic parent phase introduces an elastic energy on the interface, which is numerically unfavorable. The Khachaturyan and the Voigt/Taylor models have been implemented in the Finite Element Analysis Program FEAP and the Non-linear Material and Structure Analysis Suite Zset independently. Both yield the same results. In a preliminary test, the two interpolation approaches, the case-dependent and the analogous approach, have been tested for a varying number of phases using different linear and nonlinear interpolation functions. The analogous approach is valid for at most two phases or a linear interpolation function. That means the case-dependent approach has to be used when considering three or more 
phases while using a non-liner interpolation function. In the literature, the Khachaturyan approach here uses a linear interpolation scheme which can cause the order parameter field to pass through inadmissible values during the evolution and can lead in the worst case to an invalid solution. Different solutions can be obtained for both approaches and the same set of parameters. As shown here, a possible solution is to use a at least cubic interpolation function in the Khachaturyan approach, obtaining the same result as in the Voigt/Taylor approach. The advantage of the Voigt/Taylor approach is the flexibility. The generalization to an arbitrary number of martensitic phases is straightforward. Furthermore, different material laws for the martensitic child phases can be employed. As a downside, the phases need to be solved independently. Employing parallelization can mitigate this issue. Only a subset of the martensitic phases are present locally and changing actively. In the future, a criterion determining the active phases can be introduced. Here, only elastic deformation is considered. In the future, the model will be extended using plasticity.

Acknowledgements Funded by the Deutsche Forschungsgemeinschaft (DFG, German Research Foundation) - Project-ID 172116086 - SFB 926

\section{References}

1. Ahlers, M.: The martensitic transformation. Revista Materia 9(3), 169-183 (2004)

2. Ahn, T.H., Oh, C.S., Kim, D.H., Oh, K., Bei, H., George, E.P., Han, H.: Investigation of strain-induced martensitic transformation in metastable austenite using nanoindentation. Scripta Mater. 63(5), 540-543 (2010)

3. Aihara, S., Takaki, T., Takada, N.: Multi-phase-field modeling using a conservative Allen-Cahn equation for multiphase flow. Comput. Fluids 178, 141-151 (2019)

4. Allen, S.M., Cahn, J.W.: A microscopic theory for antiphase boundary motion and its application to antiphase domain coarsening. Acta Metall. 27(6), 1085-1095 (1979)

5. Ammar, K., Appolaire, B., Cailletaud, G., Forest, S.: Combining phase field approach and homogenization methods for modelling phase transformation in elastoplastic media. Europ. J. Comput. Mech. Revue Européenne de Mécanique Numérique 18(5-6), 485-523 (2009)

6. Ammar, K., Appolaire, B., Forest, S., Cottura, M., Le Bouar, Y., Finel, A.: Modelling inheritance of plastic deformation during migration of phase boundaries using a phase field method. Meccanica 49(11), 2699-2717 (2014)

7. Artemev, A., Jin, Y., Khachaturyan, A.: Three-dimensional phase field model of proper martensitic transformation. Acta Mater. 49(7), 1165-1177 (2001)

8. Artemev, A., Wang, Y., Khachaturyan, A.: Three-dimensional phase field model and simulation of martensitic transformation in multilayer systems under applied stresses. Acta Mater. 48(10), 2503-2518 (2000)

9. Aurich, J.C., Mayer, P., Kirsch, B., Eifler, D., Smaga, M., Skorupski, R.: Characterization of deformation induced surface hardening during cryogenic turning of aisi 347. CIRP Ann. 63(1), 65-68 (2014)

10. Bain, E.C., Dunkirk, N.: The nature of martensite. Trans. AIME 70(1), $25-47$ (1924)

11. Bhadak, B., Sankarasubramanian, R., Choudhury, A.: Phase-field modeling of equilibrium precipitate shapes under the influence of coherency stresses. Metall. Mater. Trans. A 49(11), 5705-5726 (2018)

12. Bhadeshia, H.: Martensitic transformation. Encyclopedia of materials science: Science and technology pp. 5203-5206 (2001)

13. Bhadeshia, H., Wayman, C.: Phase transformations: Nondiffusive. Phys. Metall., pp. 1021-1072. Elsevier (2014)

14. Bronsard, L., Reitich, F.: On three-phase boundary motion and the singular limit of a vector-valued ginzburg-landau equation. Arch. Rational Mech. Anal. 124(4), 355-379 (1993)

15. Cahn, J.W., Hilliard, J.E.: Free energy of a nonuniform system i interfacial free energy. J. Chem. Phys. 28(2), $258-267$ (1958)

16. Diewald, F., Kuhn, C., Heier, M., Langenbach, K., Horsch, M., Hasse, H., Müller, R.: Investigating the stability of the phase field solution of equilibrium droplet configurations by eigenvalues and eigenvectors. Comput. Mater. Sci. 141, 185-192 (2018)

17. Echebarria, B., Folch, R., Karma, A., Plapp, M.: Quantitative phase-field model of alloy solidification. Phys. Rev. E 70(6), 061604 (2004)

18. Greninger, A.B., Troiano, A.R.: The mechanism of martensite formation. Trans. AIME 185(9), 590-598 (1949)

19. Gurtin, M.E.: Generalized ginzburg-landau and cahn-hilliard equations based on a microforce balance. Phys. D Nonlinear Phenomena 92(3-4), 178-192 (1996)

20. Headley, T., Brooks, J.: A new bcc-fcc orientation relationship observed between ferrite and austenite in solidification structures of steels. Metall. Mater. Trans. A 33(1), 5-15 (2002)

21. Heida, M.: On the derivation of thermodynamically consistent boundary conditions for the cahn-hilliard-navier-stokes system. Int. J. Eng. Sci. 62, 126-156 (2013)

22. Herrmann, C., Schoof, E., Schneider, D., Schwab, F., Reiter, A., Selzer, M., Nestler, B.: Multiphase-field model of small strain elasto-plasticity according to the mechanical jump conditions. Comput. Mech. 62(6), 1399-1412 (2018)

23. Hori, M., Nemat-Nasser, S.: On two micromechanics theories for determining micro-macro relations in heterogeneous solids. Mech. Mater. 31(10), 667-682 (1999)

24. Keating, D., Goland, A.: Atomic displacements in iron martensite. Acta Metall. 15(12), 1805-1814 (1967)

25. Kelly, P.: Crystallography of martensite transformations in steels. Phase Trans. Steels, pp. 3-33. Elsevier (2012)

26. Kim, J., Lee, S., Choi, Y., Lee, S.M., Jeong, D.: Basic principles and practical applications of the cahn-hilliard equation. Math. Probl. Eng., (2016) 
27. Kim, S.A., Johnson, W.L.: Elastic constants and internal friction of martensitic steel, ferritic-pearlitic steel, and $\alpha$-iron. Mater. Sci. Eng. A 452, 633-639 (2007)

28. Klostermann, J.: The concept of the habit plane and the phenomenological theories of the martensite transformation. J. Less Common Metals 28(1), 75-94 (1972)

29. Kuhn, C., Müller, R.: A continuum phase field model for fracture. Eng. Fracture Mech. 77(18), 3625-3634 (2010)

30. Kurdjumow, G., Sachs, G.: Über den mechanismus der stahlhärtung. Zeitschrift für Physik 64(5-6), 325-343 (1930)

31. Mayer, P., Skorupski, R., Smaga, M., Eifler, D., Aurich, J.C.: Deformation induced surface hardening when turning metastable austenitic steel aisi 347 with different cryogenic cooling strategies. Procedia CIRP 14, 101-106 (2014)

32. Meiser, J., Urbassek, H.: Ferrite-to-austenite and austenite-to-martensite phase transformations in the vicinity of a cementite particle: A molecular dynamics approach. Metals 8(10), 837 (2018)

33. Mercier, O., Melton, K., Gremaud, G., Hägi, J.: Single-crystal elastic constants of the equiatomic niti alloy near the martensitic transformation. J. Appl. Phys. 51(3), 1833-1834 (1980)

34. Miehe, C., Hofacker, M., Welschinger, F.: A phase field model for rate-independent crack propagation: Robust algorithmic implementation based on operator splits. Comput. Methods Appl. Mech. Eng. 199(45-48), 2765-2778 (2010)

35. Miehe, C., Welschinger, F., Hofacker, M.: Thermodynamically consistent phase-field models of fracture: Variational principles and multi-field fe implementations. Int. J. Numer. Methods Eng. 83(10), 1273-1311 (2010)

36. Mo, K., Zhou, Z., Miao, Y., Yun, D., Tung, H.M., Zhang, G., Chen, W., Almer, J., Stubbins, J.F.: Synchrotron study on load partitioning between ferrite/martensite and nanoparticles of a 9cr ods steel. J. Nuclear Mater. 455(1-3), 376-381 (2014)

37. Mosler, J., Shchyglo, O., Hojjat, H.M.: A novel homogenization method for phase field approaches based on partial rank-one relaxation. J. Mech. Phys. Solids 68, 251-266 (2014)

38. Muench, I., Gierden, C., Wagner, W.: A phase field model for stress-based evolution of load-bearing structures. Int. J. Numer. Methods Eng. 115(13), 1580-1600 (2018)

39. Nishiyama, Z.: X-ray investigation of the mechanism of the transformation from face centered cubic lattice to body centered cubic. Sci. Rep. Tohoku Univ. 23, 637 (1934)

40. Porter, D.A., Easterling, K.E., Sherif, M.: Phase Transformations in Metals and Alloys, (Revised Reprint). CRC Press, Cambridge (2009)

41. Qin, R.S., Bhadeshia, H.K.: Phase field method. Mater. Sci. Technol. 26(7), 803-811 (2010)

42. Rancourt, Vd., Appolaire, B., Forest, S., Ammar, K.: Homogenization of viscoplastic constitutive laws within a phase field approach. J. Mech. Phys. Solids 88, 35-48 (2016)

43. Sandoval, L., Urbassek, H.M., Entel, P.: The bain versus nishiyama-wassermann path in the martensitic transformation of fe. New J. Phys. 11(10), 103027 (2009)

44. Schmidt, S., Dornisch, W., Müller, R.: A phase field model for martensitic transformation coupled with the heat equation. GAMM-Mitteilungen 40(2), 138-153 (2017)

45. Schmitt, R., Müller, R., Kuhn, C.: A phase field model for martensitic transformations. PAMM 12(1), 261-262 (2012)

46. Schmitt, R., Müller, R., Kuhn, C., Urbassek, H.M.: A phase field approach for multivariant martensitic transformations of stable and metastable phases. Arc. Appl. Mech. 83(6), 849-859 (2013)

47. Schneider, D., Schoof, E., Tschukin, O., Reiter, A., Herrmann, C., Schwab, F., Selzer, M., Nestler, B.: Small strain multiphasefield model accounting for configurational forces and mechanical jump conditions. Comput. Mech. 61(3), 277-295 (2018)

48. Schneider, D., Tschukin, O., Choudhury, A., Selzer, M., Böhlke, T., Nestler, B.: Phase-field elasticity model based on mechanical jump conditions. Comput. Mech. 55(5), 887-901 (2015)

49. Schoof, E., Schneider, D., Streichhan, N., Mittnacht, T., Selzer, M., Nestler, B.: Multiphase-field modeling of martensitic phase transformation in a dual-phase microstructure. Int. J. Solids Struct. 134, 181-194 (2018)

50. Smallman, R.E.: Modern physical metallurgy. Elsevier, Amsterdam (2016)

51. Steinbach, I., Pezzolla, F.: A generalized field method for multiphase transformations using interface fields. Physica D Nonlinear Phenomena 134(4), 385-393 (1999)

52. Steinbach, I., Pezzolla, F., Nestler, B., Seeßelberg, M., Prieler, R., Schmitz, G., Rezende, J.: A phase field concept for multiphase systems. Physica D Nonlinear Phenomena 94(3), 135-147 (1996)

53. Strangwood, M.: Fundamentals of ferrite formation in steels. In: Phase Transformations in Steels, pp. 187-224. Elsevier (2012)

54. Taylor, R., Zienkiewicz, O.C.: The finite element method. Butterworth-Heinemann, Oxford (2013)

55. Transvalor: Z-set Material \& Structure Analysis Suite (2020). http://zset-software.com

56. Vvedensky, D.D.: Diffusionless transformations. In: Transformations of Materials, 2053-2571, pp. 7-1 to 7-14. Morgan \& Claypool Publishers (2019)

57. Wang, S.L., Sekerka, R., Wheeler, A., Murray, B., Coriell, S., Braun, R., McFadden, G.: Thermodynamically-consistent phase-field models for solidification. Physica D Nonlinear Phenomena 69(1-2), 189-200 (1993)

58. Wassermann, G.: über den mechanismus der a-g umwandlung des eisens. Mitt. K-Wilh-Inst. Eisenforsch 17, 149-155 (1935)

59. Yamanaka, A., Takaki, T., Tomita, Y.: Elastoplastic phase-field simulation of self- and plastic accommodations in cubictetragonal martensitic transformation. Mater. Sci. Eng. A 491(1), 378-384 (2008)

60. Yardley, V., Payton, E.: Austenite-martensite/bainite orientation relationship: characterisation parameters and their application. Mater. Sci. Technol. 30(9), 1125-1130 (2014)

Publisher's Note Springer Nature remains neutral with regard to jurisdictional claims in published maps and institutional affiliations. 